\title{
Global Dynamics of a Virus-Immune System with Virus-Guided Therapy and Saturation Growth of Virus
}

\author{
Qian Li and Yanni Xiao \\ School of Mathematics and Statistics, Xi'an Jiaotong University, Xian 710049, China \\ Correspondence should be addressed to Yanni Xiao; yxiao@mail.xjtu.edu.cn
}

Received 2 February 2018; Revised 16 May 2018; Accepted 4 June 2018; Published 9 July 2018

Academic Editor: Carlo Cosentino

Copyright ( 2018 Qian Li and Yanni Xiao. This is an open access article distributed under the Creative Commons Attribution License, which permits unrestricted use, distribution, and reproduction in any medium, provided the original work is properly cited.

\begin{abstract}
We considered a piecewise virus-immune dynamic model to investigate the effectiveness of the HIV virus loads-guided structured treatment interruptions (STIs). To better describe the biological reality, we extended the existing models by taking the carrying capacity of the virus loads into consideration to indicate the saturated growth of virus loads. We initially investigated the sliding dynamics of the proposed model and then obtained the global dynamics of the proposed model. Our main results showed that the system can exhibit very complex and diverse dynamic behaviors including a globally asymptotically stable equilibrium, bistable equilibra, and tristable equilibria, depending on the dynamics of the subsystems and the threshold level. In particular, an interesting result indicated that, with a proper threshold condition, the virus-guided therapy policy can successfully control the virus loads far below its carrying capacity and maintain the activity of the immune system for the case that the effector cells always go to zero without therapy or with continuous therapy. The finding suggested that the optimal strategy should be individual-based due to coexistence of multiple stable steady states, depending on the threshold conditions and the initial levels of viral loads and effector cells of the patients.
\end{abstract}

\section{Introduction}

Highly active antiretroviral therapy (HAART) plays significant roles in improving survival and reducing morbidity and mortality in HIV patients [1,2]. Nevertheless, lifelong HAART confronts many complicated problems such as adherence difficulties and the evolution of drug resistance [36]. Structured treatment interruptions (STIs), the alternative strategies, have been designed to effectively achieve sustained specific immunity for early therapy in HIV infection. Indeed, STIs are beneficial for some chronically infected individuals who may need to take drugs throughout their lives as well as the reconstruction of patients' immune system when they are not taking the drugs [7].

Since the first HIV patient received the structured antiretroviral therapy in 1999 [8], STIs have been widely investigated by many researchers. Basically, it includes two kinds of treatment regimes: carrying out the therapies at fixed moments or depending on the CD4 cell counts and/or viral loads. For example, some studies designed a scheduled treatment that therapy was initiated only when the CD4 cell counts decreased below 350 cells/ $\mu l$ [9-12]. Aiming to maintain CD4 cell counts higher than 350 cells/ $\mu l$ and HIV virus loads less than 100,000 copies/ $\mu l$, Ruiz et al. designed an experiment to evaluate the safety of $\mathrm{CD} 4$ cell and HIV virusguided STIs [13]. Many works have been done to compare the STIs with the continuous antiretroviral therapy (ART), but conflicting results have been reported [7, 11, 13-17]. In particular, Maggiolo et al. made a conclusion that the structured treatment can result in the similar benefits for the patients with the continuous therapies [7], while the SMART study group found that STIs may also endanger the patients' life if their immune responses are not sufficiently stimulated [18]. STI is still an important topic since lifelong ART brings great challenges to all HIV positive individuals. Moreover, as immune therapy arises, combining the ART with immune therapy (such as interleukin-2 (IL-2) treatment) may be a good choice for treating HIV patients [19]. Modelling and quantifying the efficacy of the combination of STIs and immune therapy remains unclear and falls within the scope of this study. 
Mathematical models are useful tools for investigating the dynamics of HIV-1 infection [20, 21]. Although many studies modelled the continuous therapy for HIV-infected patients [22-24], few attempts studied the STIs through mathematical modelling. In 2000, using a standard population model, Bonhoeffer et al. argued the benefits and risk generated from STIs [25]. In the study [26], Adams et al. analyzed the efficacy of STIs with fixed moments through the optimal control theory. Further, Rosenberg et al. pointed out that it is needed to include drug resistance and CD 4 cell counts when making the structured treatment [27]. In 2006, Park et al. developed a mathematical model to investigate the impact of finite times of structured treatment guided by CD4 cell counts on HIV patients [28]. Then, Tang et al. [29] formulated a piecewise system to describe the CD4 cell-guided STIs, quantitatively explored STI strategies, and explained some controversial conclusions from different clinical studies. To further model immune therapy, Tang et al. [30] proposed a piecewise virus-immune dynamic model considering the combined antiretroviral therapy with interleukin-2 (IL-2) treatment. The model is given as follows:

$$
\begin{aligned}
& \dot{x}=r x-p x y-\varepsilon_{1} \Phi x, \\
& \dot{y}=\frac{c x y}{1+\omega x}-q x y-\delta y+\varepsilon_{2} \Phi y,
\end{aligned}
$$

with

$$
\Phi= \begin{cases}0, & \text { if } H(x)=x-V_{T}<0, \\ 1, & \text { if } H(x)=x-V_{T}>0,\end{cases}
$$

where $x$ and $y$ represent the HIV virus loads and the effector cells, respectively. Note that a critical value of virus loads, denoted by $V_{T}$, is set to trigger the combined therapy. That is, only when the virus loads exceed the threshold, we carry out the antiretroviral therapy and immune therapy (such as the interleukin-2 (IL-2) treatment) simultaneously for the patients, with $\varepsilon_{1}$ being the elimination rate of HIV virus due to antiretroviral therapy and $\varepsilon_{2}$ representing the growth rate of the effector cells due to immune therapy. Here, $r$ is the growth rate of HIV virus which incorporates both their multiplication and death, $\delta$ is the death rate of the effector cells, $p$ denotes the rate of binding of the effector cells to the virus loads, $q$ represents the rate of inactivation of the effector cells, and $c x /(1+\omega x)$ denotes that effector cell multiplication due to immune response has a maximum value as the HIV virus loads become large sufficiently.

In [30], the authors mainly discussed the global dynamics of system (1), and they further considered the efficacy of the effector cell-guided or both the effector cell and the HIV virus-guided piecewise therapies [31, 32]. Their results indicated that HIV virus can be controlled under some conditions with virus or effector cell-guided therapy, but still there are some parameter regions that the threshold policy can not prevent HIV from growing infinitely. As we can see from model (1), they simply assumed the linear growth of HIV virus. However, this is not what the thing looks like. Some clinical facts indicate that there should be "saturation effects" for the growth of HIV virus [33, 34]. The linear growth of HIV virus is unreasonable. Note that including the saturated growth of the HIV virus makes the model more natural but significantly changes the dynamics of the basic immune-virus system. Little is known about whether HIV virus-guided therapy based on this more natural description of its growth can completely control the growth of HIV and falls within the scope of this study.

The main purpose of this paper is to propose a piecewise model with the saturated growth for virus loads to describe HIV virus-guided therapy and then evaluate the effectiveness of the threshold policy through analyzing the global dynamics of the proposed model. The remaining parts of this paper are organized as follows. In Section 2, we initially propose the model, give some basic definitions of the Filippov system and also briefly conclude the dynamic behaviors of two subsystems. In Section 3, we mainly discuss the sliding mode and sliding dynamics, including the existence of the sliding domain and the pseudoequilibrium. In Section 4, we investigate the global dynamics of the proposed model. Finally, we provide the discussion and some biological conclusions.

\section{Filippov Model and Preliminaries}

In this paper, we extend model (1) by including the saturated growth of HIV virus, and hence we propose the following model:

$$
\begin{aligned}
& \dot{x}=r x(1-\eta x)-p x y-\varepsilon_{1} \Phi x, \\
& \dot{y}=\frac{c x y}{1+\omega x}-q x y-\delta y+\varepsilon_{2} \Phi y,
\end{aligned}
$$

with

$$
\Phi= \begin{cases}0, & \text { if } H(x)=x-V_{T}<0 \\ 1, & \text { if } H(x)=x-V_{T}>0\end{cases}
$$

Here, $1 / \eta$ represents the carrying capacity of virus loads. Letting $Z=(x, y)^{T}$

$$
\begin{aligned}
& F_{R_{1}}(Z)=\left(r x(1-\eta x)-p x y, \frac{c x y}{1+\omega x}-q x y-\delta y\right)^{T} \\
& F_{R_{2}}(Z)=\left(r x(1-\eta x)-p x y-\varepsilon_{1} x, \frac{c x y}{1+\omega x}-q x y\right. \\
& \left.\quad-\delta y+\varepsilon_{2} y\right)^{T} .
\end{aligned}
$$

Then system (3) with (4) can be rewritten as the following general planer Filippov system [35-37]:

$$
Z(t)= \begin{cases}F_{R_{1}}(Z), & Z \in R_{1}, \\ F_{R_{2}}(Z), & Z \in R_{2} .\end{cases}
$$

with $R_{1}=\left\{Z \in R_{+}^{2} \mid H(Z)<0\right\}$ and $R_{2}=\left\{Z \in R_{+}^{2} \mid H(Z)>\right.$ $0\}$. Additionally, we describe the discontinuity boundary $\Sigma$ separating the two regions $R_{1}$ and $R_{2}$ as $\Sigma=\{Z \in$ $\left.R_{+}^{2} \mid H(Z)=0\right\}$, where $H(Z)$ is a smooth scale function 
nonvanishing on $\Sigma$. For convenience, we denote Filippov system (6) defined in region $R_{1}$ as system $S_{1}$ and that defined in region $R_{2}$ as system $S_{2}$. Then we let

$$
\sigma(Z)=\left\langle H_{Z}(Z), F_{R_{1}}(Z)\right\rangle\left\langle H_{Z}(Z), F_{R_{2}}(Z)\right\rangle,
$$

where $\langle\cdot\rangle$ represents the standard scalar product and $H_{Z}(Z)=$ $(1,0)^{T}$ denotes the nonvanishing gradient of $H$ on $\Sigma$. In the following, we replace $\left\langle H_{Z}(Z), F_{R_{i}}(Z)\right\rangle$ with the notation $F_{R_{i}} H(Z)$ for $i=1,2$.

In order to consider the trajectory of the Filippov vector field $\left(F_{R_{1}}(Z), F_{R_{2}}(Z)\right)$, through a point $Z \in \Sigma$, we distinguish the following regions on $\Sigma$ according to whether or not the vector field points towards it:

(a) Crossing region:

$$
\Sigma_{c}=\left\{Z \in \Sigma \mid F_{R_{1}} H(Z) \cdot F_{R_{2}} H(Z)>0\right\},
$$

(b) Sliding region:

$$
\Sigma_{s}=\left\{Z \in \Sigma \mid F_{R_{1}} H(Z)>0, F_{R_{2}} H(Z)<0\right\},
$$

(c) Escaping region:

$$
\Sigma_{e}=\left\{Z \in \Sigma \mid F_{R_{1}} H(Z)<0, F_{R_{2}} H(Z)>0\right\} .
$$

Further, we can define a scalar differential equation on the sliding domain by employing the Filippov method. Let

$$
g(Z)=\lambda F_{R_{1}}+(1-\lambda) F_{R_{2}}, \quad \lambda=\frac{\left\langle H_{Z}, F_{R_{2}}\right\rangle}{\left\langle H_{Z}, F_{R_{2}}-F_{R_{1}}\right\rangle}
$$

with $0<\lambda<1$. Then, we have that

$$
Z^{\prime}=g(Z), \quad Z \in \Sigma_{s}
$$

describes the dynamics of system (6) on the sliding segment $\Sigma_{s}$. In what follows, we give the definitions of two types of equilibria of Filippov system (6).

Definition 1. A point $Z^{*}$ is referred to as a real equilibrium of system (6) if $F_{R_{1}}\left(Z^{*}\right)=0, H\left(Z^{*}\right)<0$, or $F_{R_{2}}\left(Z^{*}\right)=0$, $H\left(Z^{*}\right)>0$. A point $Z^{*}$ is called a virtual equilibrium of system (6) if $F_{R_{1}}\left(Z^{*}\right)=0, H\left(Z^{*}\right)>0$, or $F_{R_{2}}\left(Z^{*}\right)=0, H\left(Z^{*}\right)<$ 0 . Both the real equilibrium and virtual equilibrium are called regular equilibria.

Definition 2. A point $Z^{*}$ is referred to as a pseudoequilibrium if it is an equilibrium of the sliding mode of system (6); i.e., the equilibrium of the equation $Z^{\prime}=g(Z)=0$ satisfies $H\left(Z^{*}\right)=$ 0 .

Before exploring the global dynamics of the Filippov system (3), it is essential to have a clear view of the dynamics of two basic subsystems. Thus, we first briefly make some conclusions on the dynamics of subsystem $S_{1}$ and subsystem $\mathrm{S}_{2}$.

For subsystem $S_{1}$, there always exists a trivial equilibrium $E_{10}=(0,0)$ and a boundary equilibrium $E_{1 \eta}=\left(x_{1 \eta}, 0\right)=$ $(1 / \eta, 0)$. The positive equilibrium $\left(x^{*}, y^{*}\right)$ of subsystem $S_{1}$ satisfies the following equations:

$$
\begin{aligned}
& r\left(1-\eta x^{*}\right)-p y^{*}=0, \\
& \frac{c x^{*}}{1+\omega x^{*}}-q x^{*}-\delta=0 .
\end{aligned}
$$

Let

$$
\begin{aligned}
f(x) & \doteq c x-(q x+\delta)(1+\omega x) \\
& =-q \omega x^{2}+(c-q-\delta \omega) x-\delta,
\end{aligned}
$$

and $\Delta_{1}=c-q-\delta \omega-2 \sqrt{q \omega \delta}$. It is easy to verify that if $\Delta_{1}>0$ holds true, then $f(x)=0$ has two positive roots which are given as

$$
\begin{aligned}
& x_{11}=\frac{c-q-\delta \omega-\sqrt{(c-q-\delta \omega)^{2}-4 q \omega \delta}}{2 q \omega}, \\
& x_{12}=\frac{c-q-\delta \omega+\sqrt{(c-q-\delta \omega)^{2}-4 q \omega \delta}}{2 q \omega} .
\end{aligned}
$$

Particularly, we know $f(x)>0$ for $x \in\left(x_{11}, x_{12}\right)$ and $f(x)<$ 0 for $x \in\left(0, x_{11}\right)$ or $x \in\left(x_{12},+\infty\right)$. Substituting $x_{11}$ or $x_{12}$ into the first equation of (13), we get

$$
\begin{aligned}
& y_{11}=\frac{r}{p}\left(1-\eta x_{11}\right), \\
& y_{12}=\frac{r}{p}\left(1-\eta x_{12}\right) .
\end{aligned}
$$

Thus, if

$$
\begin{aligned}
\frac{c-q-\delta \omega \mp \sqrt{(c-q-\delta \omega)^{2}-4 q \omega \delta}}{2 q \omega} & <\frac{1}{\eta} \\
\left(\text { i.e., } x_{1 i}\right. & \left.<\frac{1}{\eta}, i=1,2\right)
\end{aligned}
$$

holds true, then $E_{1 i}\left(x_{1 i}, y_{1 i}\right), i=1,2$, are positive equilibria of system $S_{1}$.

Next, we briefly investigate the stability of the equilibria of system $S_{1} . E_{1 \eta}$ is locally stable if $(c / \eta) /(1+\omega / \eta)-q / \eta-\delta<0$, which is equivalent to $\Delta_{1}<0$ or $\Delta_{1}>0, y_{11}<0$ or $\Delta_{1}>0$, and $y_{12}>0$. Define a function $K(x, y)=1 / x y$, and calculate

$$
L=\frac{\partial}{\partial x}\left(K(x, y) \frac{d x}{d t}\right)+\frac{\partial}{\partial y}\left(K(x, y) \frac{d y}{d t}\right)=-\frac{r \eta}{y},
$$

where $d x / d t$ and $d y / d t$ are defined in system $S_{1}$. Since all the parameters are positive and solutions to system $S_{1}$ initiating from $R_{+}^{2}$ are nonnegative, $L$ does not change its sign in the first quadrant. Hence according to Dulac-Bendixson criterion, we get that there are no limit cycles or homoclinic connections for system $S_{1}$. Therefore, if there is no positive equilibrium, then the boundary equilibrium $E_{1 \eta}$ is globally asymptotically stable because system $S_{1}$ is bounded. Further, if $\Delta_{1}>0$ and $x_{11}<1 / \eta<x_{12}$ hold true, it is easy to verify that $f(1 / \eta)>$ 0 , and hence the boundary equilibrium $E_{1 \eta}$ is unstable. Therefore, under this scenario, positive equilibrium $E_{11}$ is 
globally asymptotically stable. When there are two positive equilibria of system $S_{1}$, the dynamics of system $S_{1}$ have been shown in [38] that the equilibrium $E_{12}$ is always an unstable saddle and $E_{11}$ and $E_{1 \eta}$ are bistable. Then the dynamics of system $S_{1}$ can be concluded as follows.

Proposition 3. System $S_{1}$ always has a trivial equilibrium $E_{10}$ and a boundary equilibrium $E_{1 \eta}$. If $\Delta_{1}<0$ or $\Delta_{1}>0$ and $x_{11}>1 / \eta$ hold true, then there is no positive equilibrium, and the boundary equilibrium $E_{1 \eta}$ is globally asymptotically stable; If $\Delta_{1}>0$ and $x_{11}<1 / \eta<x_{12}$, there is one and only one positive equilibrium $E_{11}$ which is globally asymptotically stable; If $\Delta_{1}>0$ and $x_{12}<1 / \eta$, there are two positive equilibria $E_{11}$ and $E_{12}$, and $E_{11}$ and $E_{1 \eta}$ are bistable.

If $r>\varepsilon_{1}$ and $\delta>\varepsilon_{2}$, system $S_{2}$ has similar properties to system $S_{1}$. Denote $\Delta_{2}=c-q-\left(\delta-\varepsilon_{2}\right) \omega-2 \sqrt{q \omega\left(\delta-\varepsilon_{2}\right)}$ and

$$
\begin{aligned}
& x_{2 i} \\
& =\frac{c-q-\left(\delta-\varepsilon_{2}\right) \omega \mp \sqrt{\left(c-q-\left(\delta-\varepsilon_{2}\right) \omega\right)^{2}-4 q \omega\left(\delta-\varepsilon_{2}\right)}}{2 q \omega}, \\
& y_{2 i}=\frac{r}{p}\left(1-\eta x_{2 i}\right)-\frac{\varepsilon_{1}}{p},
\end{aligned}
$$

Similarly, if

$$
\begin{gathered}
\frac{c-q-\left(\delta-\varepsilon_{2}\right) \omega \mp \sqrt{\left(c-q-\left(\delta-\varepsilon_{2}\right) \omega\right)^{2}-4 q \omega\left(\delta-\varepsilon_{2}\right)}}{2 q \omega} \\
<\frac{r-\varepsilon_{1}}{r \eta} \quad\left(\text { i.e., } x_{2 i}<\frac{r-\varepsilon_{1}}{r \eta}, i=1,2\right)
\end{gathered}
$$

holds true, then $E_{2 i}\left(x_{2 i}, y_{2 i}\right), i=1,2$, are positive equilibria of system $S_{2}$. Then we can conclude the dynamics of system $S_{2}$ as follows.

Proposition 4. System $S_{2}$ has a trivial equilibrium $E_{20}=$ $(0,0)$ and a boundary equilibrium $E_{2 \eta}=\left(x_{2 \eta}, 0\right)=((r-$ $\left.\left.\varepsilon_{1}\right) / r \eta, 0\right)$. If $\Delta_{2}<0$ or $\Delta_{2}>0$ and $x_{21}>\left(r-\varepsilon_{1}\right) / r \eta$, system $S_{2}$ has no positive equilibrium, and the boundary equilibrium $E_{2 \eta}$ is globally asymptotically stable; if $\Delta_{2}>0$ and $x_{22}<$ $\left(r-\varepsilon_{1}\right) / r \eta<x_{21}$ hold true, there is one and only one positive equilibrium $E_{21}$ of system $S_{2}$ which is globally asymptotically stable; if $\Delta_{2}>0$ and $x_{22}<\left(r-\varepsilon_{1}\right) / r \eta$ hold true, there are two positive equilibria $E_{21}$ and $E_{22}$, and both $E_{21}$ and $E_{2 \eta}$ are bistable.

\section{Sliding Dynamics}

According to the definition of $\sigma(Z)$ in Section 2, we have

$\sigma(Z)$

$$
=(r x(1-\eta x)-p x y)\left(r x(1-\eta x)-p x y-\varepsilon_{1} x\right) .
$$

Let $\sigma(Z)<0$, and one yields

$$
\frac{r}{p}(1-\eta x)-\frac{\varepsilon_{1}}{p}<y<\frac{r}{p}(1-\eta x) .
$$

It is reasonable to assume that the therapy should be done before virus growing and approaching its carrying capacity; therefore, in this study, we always assume that $V_{T}<1 / \eta$ holds true. Then, if $\left(1-\varepsilon_{1} / r\right) / \eta<V_{T}<1 / \eta$ (i.e., $\left.x_{2 \eta}<V_{T}<x_{1 \eta}\right)$, the sliding segment is $\Sigma_{s}^{1}=\left\{(x, y) \mid x=V_{T}, 0 \leq y \leq\right.$ $\left.(r / p)\left(1-\eta V_{T}\right)\right\}$; if $0<V_{T}<x_{2 \eta}$, then the sliding segment is $\Sigma_{s}^{2}=\left\{x, y \mid x=V_{T},(r / p)\left(1-\eta V_{T}\right)-\varepsilon_{1} / p \leq y \leq(r / p)(1-\right.$ $\left.\left.\eta V_{T}\right)\right\}$.

Next, we employ Utkin's equivalent control method introduced in [39] to examine the sliding dynamics in the region $\Sigma_{s}$. It follows from $H(x)=0$ and the first equation of system (3) that

$$
\frac{d H}{d t}=\frac{d x}{d t}=r x(1-\eta x)-p x y-\Phi \varepsilon_{1} x=0 .
$$

Solving (23) with respect to $\Phi$ yields

$$
\Phi=\frac{r}{\varepsilon_{1}}(1-\eta x)-\frac{p}{\varepsilon_{1}} y .
$$

Then substituting $\Phi$ into the second equation of system (3) gives

$$
\begin{aligned}
& \frac{d y}{d t} \\
& =y\left(-\frac{\varepsilon_{2}}{\varepsilon_{1}} p y+\frac{\varepsilon_{2}}{\varepsilon_{1}} r\left(1-\eta V_{T}\right)+\frac{c V_{T}}{1+\omega V_{T}}-q V_{T}-\delta\right) \\
& \triangleq Q_{s}\left(V_{T}, y\right) .
\end{aligned}
$$

Then, the sliding dynamics of system (3) can be described as (25). Let $Q_{s}\left(V_{T}, y\right)=0$, and we obtain two roots

$$
\begin{aligned}
& y_{0}=0, \\
& y_{p}=\frac{r}{p}\left(1-\eta V_{T}\right)+\frac{\varepsilon_{1}}{\varepsilon_{2} p}\left(\frac{c V_{T}}{1+\omega V_{T}}-q V_{T}-\delta\right) .
\end{aligned}
$$

This indicates that there are two possible pseudoequilibria $E_{0}=\left(V_{T}, 0\right)$ and $E_{p}=\left(V_{T}, y_{p}\right)$. In order to verify whether they are located at the sliding segment, we consider two situations $0<V_{T}<x_{2 \eta}$ and $x_{2 \eta}<V_{T}<x_{1 \eta}$.

It is easy to see that $E_{0}$ can not be a pseudoequilibrium when $0<V_{T}<x_{2 \eta}$, while it is always a pseudoequilibrium for $x_{2 \eta}<V_{T}<x_{1 \eta}$. Thus, we only need to analyze the existence of $E_{p}$. First, we prove the following lemmas.

Lemma 5. If $\Delta_{1}>0$ and $\Delta_{2}>0$ hold true, then we have $x_{21}<x_{11}<x_{12}<x_{22}$.

Proof. Let

$$
g_{1}(z)=c-q-\omega z-\sqrt{(c-q-\omega z)^{2}-4 q \omega z},
$$

(with $z>0$ ). 
To ensure the function to be well defined, we need $c-q-\omega z>$ $2 \sqrt{q \omega z}$ (i.e., $\Delta_{1}>0$ ). Further, we know

$$
\begin{aligned}
c-q-\omega z & >2 \sqrt{q \omega z} \Longleftrightarrow \\
c-q-\omega z & >0, \\
(c-q-\omega z)^{2}-4 q \omega z & >0 \Longleftrightarrow \\
z & <\frac{c-q}{\omega}, \\
0 & <z<\frac{c+q-2 \sqrt{q c}}{\omega} \\
\text { or } z & >\frac{c+q+2 \sqrt{q c}}{\omega} .
\end{aligned}
$$

Definitely, we have $(c-q) / \omega<(c+q+2 \sqrt{q c}) / \omega$, and $(c-q) / \omega>$ $(c+q-2 \sqrt{q c}) / \omega$ hold true. Therefore, the domain for $g_{1}(z)$ is $(0,(c+q-2 \sqrt{q c}) / \omega)$, which is a continuous interval. Through simple calculation, we obtain

$$
g_{1}^{\prime}(z)=-\omega+\omega \frac{c+q-\omega z}{\sqrt{(c-q-\omega z)^{2}-4 q \omega z}} .
$$

Then, we have

$$
\begin{gathered}
g_{1}^{\prime}(z)>0 \Longleftrightarrow \\
c+q-\omega z>\sqrt{(c-q-\omega z)^{2}-4 q \omega z} \Longleftrightarrow \\
4 q c>0 .
\end{gathered}
$$

Because all the parameters are positive, $g_{1}^{\prime}(z)>0$ always holds true in its domain. Then, according to $\Delta_{1}>0, \Delta_{2}>0$, and $\delta>\delta-\varepsilon_{2}>0$, we have $x_{21}<x_{11}$. Similarly, let

$$
g_{2}(z)=c-q-\omega z+\sqrt{(c-q-\omega z)^{2}-4 q \omega z} .
$$

which is also defined in the interval $0<z<(c-q-2 \sqrt{q c}) / \omega$. Note that $g_{2}^{\prime}(z)<0$ is equivalent to $c+q-\omega z>0$. Further, it is obvious that $c+q-\omega z>c-q-\omega z>0$. This means that $g_{2}^{\prime}(z)<$ 0 always holds true in its domain. Thus, we obtain that $x_{12}<$ $x_{22}$ because $\Delta_{1}>0, \Delta_{2}>0$ and $\delta>\delta-\varepsilon_{1}>0$. In conclusion, we have $x_{21}<x_{11}<x_{12}<x_{22}$ if $\Delta_{1}>0$ and $\Delta_{2}>0$. The proof is completed.

Lemma 6. If $\Delta_{1}<0$ (or $\Delta_{2}<0$ ) holds true, then we have $y_{p}<(r / p)\left(1-\eta V_{T}\right)$ (or $\left.y_{p}<(r / p)\left(1-\eta V_{T}\right)-\varepsilon_{1} / p\right)$. If $\Delta_{1}>0$ and $\Delta_{2}>0$, then we have that $(r / p)\left(1-\eta V_{T}\right)-\varepsilon_{1} / p<y_{p}<$ $(r / p)\left(1-\eta V_{T}\right)$ for $x_{21}<V_{T}<x_{11}$ or $x_{12}<V_{T}<x_{22} ; y_{p}<$ $(r / p)\left(1-\eta V_{T}\right)-\varepsilon_{1} / p$ for $0<V_{T}<x_{21}$ or $V_{T}>x_{22}$; and $y_{p}>$ $(r / p)\left(1-\eta V_{T}\right)$ for $x_{11}<V_{T}<x_{12}$.

Proof. Let $f_{1}(x)=c x /(1+\omega x)-q x-\delta$, and we know that $f_{1}(x)<0$ if $\Delta_{1}<0$. Therefore, it follows from the formula of $y_{p}$ that when $\Delta_{1}<0$, then $y_{p}<(r / p)\left(1-\eta V_{T}\right)$. If $\Delta_{1}>0$, we get $f_{1}(x)>0$ for $x_{11}<x<x_{12}$, and $f_{1}(x)<0$ for $0<x<$ $x_{11}$ or $x>x_{12}$. This means that, when $\Delta_{1}>0$, we get $y_{p}>$ $(r / p)\left(1-\eta V_{T}\right)$ for $x_{11}<V_{T}<x_{12}$, and $y_{p}<(r / p)\left(1-\eta V_{T}\right)$ for $0<V_{T}<x_{11}$ or $V_{T}>x_{12}$. Rearranging the formula of $y_{p}$ yields

$$
\begin{aligned}
y_{p}= & \frac{r}{p}\left(1-\eta V_{T}\right)-\frac{\varepsilon_{1}}{p} \\
& +\frac{\varepsilon_{1}}{\varepsilon_{2} p}\left(\frac{c V_{T}}{1+\omega V_{T}}-q V_{T}-\delta+\varepsilon_{2}\right) .
\end{aligned}
$$

Therefore, applying similar analysis we have that when $\Delta_{2}<$ 0 , then $y_{p}<(r / p)\left(1-\eta V_{T}\right)-\varepsilon_{1} / p$; when $\Delta_{2}>0$, then $y_{p}>$ $(r / p)\left(1-\eta V_{T}\right)-\varepsilon_{1} / p$ for $x_{21}<V_{T}<x_{22}$, and $y_{p}<(r / p)(1-$ $\left.\eta V_{T}\right)-\varepsilon_{1} / p$ for $0<V_{T}<x_{21}$ or $V_{T}>x_{22}$. This completes the proof.

Lemma 7. If $\Delta_{1}>0$ and $\Delta_{2}>0$, then we have $y_{p}>\min \left\{y_{21}\right.$, $\left.y_{11}\right\}$ for $x_{21}<V_{T}<x_{11}$.

Proof. Taking the derivative of $y_{p}$ with respect to $V_{T}$ gives

$$
\frac{d y_{p}}{d V_{T}}=-\frac{r}{p} \eta+\frac{\varepsilon_{1}}{\varepsilon_{2} p}\left(\frac{c}{\left(1+\omega V_{T}\right)^{2}}-q\right) .
$$

Let $\left(d y_{p} / d V_{T}\right)>0$, we have $-(1 / \omega) \sqrt{c \varepsilon_{1} /\left(r \eta \varepsilon_{2}+q \varepsilon_{1}\right)}-1 / \omega<$ $V_{T}<(1 / \omega) \sqrt{c \varepsilon_{1} /\left(r \eta \varepsilon_{2}+q \varepsilon_{1}\right)}-1 / \omega$.

Thus, if $(1 / \omega) \sqrt{c \varepsilon_{1} /\left(r \eta \varepsilon_{2}+q \varepsilon_{1}\right)}-1 / \omega>0, y_{p}\left(V_{T}\right)$ is increasing for $0<V_{T}<(1 / \omega) \sqrt{c \varepsilon_{1} /\left(r \eta \varepsilon_{2}+q \varepsilon_{1}\right)}-1 / \omega$ and decreasing for $V_{T}>(1 / \omega) \sqrt{c \varepsilon_{1} /\left(r \eta \varepsilon_{2}+q \varepsilon_{1}\right)}-1 / \omega$; if (1/ $\omega) \sqrt{c \varepsilon_{1} /\left(r \eta \varepsilon_{2}+q \varepsilon_{1}\right)}-1 / \omega<0$, then $y_{p}\left(V_{T}\right)$ is decreasing for $V_{T}>0$. Therefore, when $x_{11}<V_{T}<x_{21}$, there may be three cases: (a) $y_{p}\left(V_{T}\right)$ is always decreasing as $V_{T}$ increases, which means $y_{p}>y_{p}\left(x_{11}\right)=y_{11}$; (b) $y_{p}\left(V_{T}\right)$ is always increasing as $V_{T}$ increases, then $y_{p}>y_{p}\left(x_{21}\right)=y_{21}$; (c) $y_{p}\left(V_{T}\right)$ is initially increasing and then decreasing as $V_{T}$ increases, and hence $y_{p}>\min \left\{y_{p}\left(x_{21}\right), y_{p}\left(x_{11}\right)\right\}=\min \left\{y_{21}, y_{11}\right\}$. This completes the proof.

It is easy to verify that if $\Delta_{2}<0$, then we have $\Delta_{1}<$ 0 , which means that there can not be $\Delta_{1}>0$ when $\Delta_{2}<0$. According to the existence of possible equilibria of subsystems $S_{1}$ or $S_{2}$, we consider the following cases to discuss the existence of the pseudoequilibrium $E_{p}$.

Case A1. There is no positive equilibrium for subsystem $S_{2}$. Then, we have three subcases:

(i) There is no positive equilibrium for subsystem $S_{1}$ (denoted by Case A11). Hence, there could be

$$
\begin{aligned}
\Delta_{1} & <0, \\
\Delta_{2} & <0 ; \\
\text { or } \Delta_{1} & <0, \\
\Delta_{2} & >0, \\
x_{21} & >\frac{\left(r-\varepsilon_{1}\right)}{r \eta} ;
\end{aligned}
$$




$$
\text { or } \begin{aligned}
\Delta_{1} & >0, \\
x_{11} & >\frac{1}{\eta}, \\
\Delta_{2} & >0 \\
x_{21} & >\frac{\left(r-\varepsilon_{1}\right)}{r \eta} .
\end{aligned}
$$

It follows from Lemma 6 that for Case $A 11$ there is always $y_{p}<(r / p)\left(1-\eta V_{T}\right)-\varepsilon_{1} / p$ for $0<V_{T}<x_{2 \eta}$. This means that there is no pseudoequilibrium for $0<V_{T}<x_{2 \eta}$. Then, we mainly consider $x_{2 \eta}<V_{T}<x_{1 \eta}$. Now, there is $(r / p)\left(1-\eta V_{T}\right)-$ $\varepsilon_{1} / p<0$. Therefore, we have $y_{p}<(r / p)\left(1-\eta V_{T}\right)-\varepsilon_{1} / p<0$ for the first subcase of Case $A 11$ (i.e., $\Delta_{1}<0, \Delta_{2}<0$ ), which means $E_{p}$ is not feasible. For the other two subcases, if $x_{21}>$ $x_{1 \eta}, E_{p}$ is not feasible as well. However, if $x_{2 \eta}<x_{21}<x_{1 \eta}$, then the sign of $y_{p}$ is not determined for the whole interval $x_{2 \eta}<V_{T}<x_{1 \eta}$. We denote

$$
\begin{array}{r}
\left(H_{1}\right): \frac{r}{p}\left(1-\eta V_{T}\right)+\frac{\varepsilon_{1}}{\varepsilon_{2} p}\left(\frac{c V_{T}}{1+\omega V_{T}}-q V_{T}-\delta\right)>0 \\
\quad\left(\text { i.e., } y_{p}>0\right) ; \\
\left(H_{2}\right): \frac{r}{p}\left(1-\eta V_{T}\right)+\frac{\varepsilon_{1}}{\varepsilon_{2} p}\left(\frac{c V_{T}}{1+\omega V_{T}}-q V_{T}-\delta\right)<0 \\
\quad\left(\text { i.e., } y_{p}<0\right) .
\end{array}
$$

Therefore, $E_{p}$ is feasible for $\left(H_{1}\right)$, while it is not feasible for $\left(\mathrm{H}_{2}\right)$.

(ii) There is a unique equilibrium for subsystem $S_{1}$ (denoted by Case A12); that is,

$$
\begin{aligned}
& \Delta_{1}>0, \\
& x_{11}<\frac{1}{\eta}<x_{12}, \\
& \Delta_{2}>0, \\
& x_{21}>\frac{\left(r-\varepsilon_{1}\right)}{r \eta} .
\end{aligned}
$$

For such case, there is no pseudoequilibrium for $0<V_{T}<$ $x_{2 \eta}$. There are two pseudoequilibria $E_{0}$ and $E_{p}$ for $\left(H_{1}\right)$ and $x_{21}<V_{T}<x_{11}$, and there is only one pseudoequilibrium $E_{0}$ for $x_{2 \eta}<V_{T}<x_{21}$ or $x_{11}<V_{T}<x_{1 \eta}$ or $\left(H_{2}\right)$ and $x_{21}<V_{T}<$ $x_{11}$.

(iii) There are two equilibria for subsystem $S_{1}$ (denoted by Case $A 13)$; that is,

$$
\begin{aligned}
& \Delta_{1}>0, \\
& x_{12}<\frac{1}{\eta}, \\
& \Delta_{2}>0, \\
& x_{21}>\frac{\left(r-\varepsilon_{1}\right)}{r \eta} .
\end{aligned}
$$

Similar to Case $A 12$, there is no pseudoequilibrium for $0<$ $V_{T}<x_{2 \eta}$. $E_{p}$ is feasible for $\left(H_{1}\right), x_{21}<V_{T}<x_{11}$, or $\left(H_{1}\right)$, $x_{12}<V_{T}<\min \left\{x_{1 \eta}, x_{22}\right\}$.

Case A2. There is one positive equilibrium for subsystem $S_{2}$. Then, consider the following:

(i) There is no positive equilibrium for subsystem $S_{1}$ (denoted by Case A21). Then, there should be

$$
\begin{aligned}
& \Delta_{1}<0, \\
& \Delta_{2}>0, \\
& x_{21}<\frac{\left(r-\varepsilon_{1}\right)}{r \eta}<x_{22} ; \\
& \text { or } \Delta_{1}>0, \\
& x_{11}>\frac{1}{\eta}, \\
& \Delta_{2}>0, \\
& x_{21}<\frac{\left(r-\varepsilon_{1}\right)}{r \eta}<x_{22} .
\end{aligned}
$$

It follows from Lemma 6 that if $\Delta_{1}<0$ or $\Delta_{1}>0, y_{11}<0$, then we have $y_{p}<(r / p)\left(1-\eta V_{T}\right)$ for $0<V_{T}<x_{1 \eta}$. Further, because $\Delta_{2}>0, y_{22}<0<y_{21}$, according to Lemma 6 , there is no pseudoequilibrium for $0<V_{T}<x_{21}$ while $E_{p}$ is feasible for $x_{21}<V_{T}<x_{2 \eta}$. Similarly, for $x_{2 \eta}<V_{T}<x_{1 \eta}$, the sign of $y_{p}$ can not be determined. Thus, when $x_{2 \eta}<V_{T}<x_{1 \eta}$, there might be either two pseudoequilibria $E_{0}$ and $E_{p}$ for $\left(H_{1}\right)$, or one pseudoequilibrium $E_{0}$ for $\left(H_{2}\right)$. Especially, if $x_{22}<x_{1 \eta}$, then $E_{p}$ is not feasible for $x_{22}<V_{T}<x_{1 \eta}$.

(ii) There is a unique positive equilibrium for subsystem $S_{1}$ (denoted by Case $A 22$ ); that is,

$$
\begin{aligned}
& \Delta_{1}>0, \\
& x_{11}<\frac{1}{\eta}<x_{12}, \\
& \Delta_{2}>0, \\
& x_{21}<\frac{\left(r-\varepsilon_{1}\right)}{r \eta}<x_{22} .
\end{aligned}
$$

For such case, it follows from Lemmas 6 and 7 that $E_{p}$ is feasible for $x_{21}<V_{T}<x_{11}$. Especially, if $x_{11}>x_{2 \eta}$, then there are two pseudoequilibria $E_{0}$ and $E_{p}$ for $x_{2 \eta}<V_{T}<x_{11}$, and there is no pseudoequilibrium for $0<V_{T}<x_{21}$.

(iii) There are two positive equilibria for subsystem $S_{1}$ (denoted by Case A23); that is,

$$
\begin{aligned}
& \Delta_{1}>0, \\
& x_{12}<\frac{1}{\eta},
\end{aligned}
$$




$$
\begin{aligned}
& \Delta_{2}>0, \\
& x_{21}<\frac{\left(r-\varepsilon_{1}\right)}{r \eta}<x_{22} .
\end{aligned}
$$

Here, we consider three subcases: $x_{12}<x_{2 \eta}, x_{11}<x_{2 \eta}<x_{12}$, or $x_{11}>x_{2 \eta}$ in terms of the relationship of $x_{2 \eta}$ to $x_{11}$ and $x_{12}$. If $x_{12}<x_{2 \eta}$, then $E_{p}$ is feasible for $x_{21}<V_{T}<x_{11}$ or $x_{12}<V_{T}<x_{2 \eta}$ or $\left(H_{1}\right)$ and $x_{2 \eta}<V_{T}<\min \left\{x_{22}, x_{1 \eta}\right\}$. If $x_{11}<x_{2 \eta}<x_{12}$, then $E_{p}$ is feasible for $x_{21}<V_{T}<x_{11}$ or $\left(H_{1}\right)$ and $x_{12}<V_{T}<\min \left\{x_{22}, x_{1 \eta}\right\}$. If $x_{11}>x_{2 \eta}$, then it follows from Lemma 7 that $E_{p}$ is feasible for $x_{21}<V_{T}<x_{11}$ or $\left(H_{1}\right)$ and $x_{12}<V_{T}<\min \left\{x_{22}, x_{1 \eta}\right\}$.

Case A3. There are two positive equilibria for subsystem $S_{2}$. Under this case, there can not be a unique positive equilibrium for subsystem $S_{1}$ according to Lemma 5. Then, consider the following:

(i) There is no positive equilibrium for subsystem $S_{1}$ (denoted by Case A31); that is,

$$
\begin{aligned}
& \Delta_{1}<0, \\
& \Delta_{2}>0, \\
& x_{22}<\frac{\left(r-\varepsilon_{1}\right)}{r \eta} .
\end{aligned}
$$

It follows from Lemma 6 that $E_{p}$ is feasible for $x_{21}<V_{T}<$ $x_{22}$.

(ii) There are two positive equilibria for subsystem $S_{1}$ (denoted by Case A32); that is,

$$
\begin{aligned}
& \Delta_{1}>0, \\
& x_{12}<\frac{1}{\eta}, \\
& \Delta_{2}>0, \\
& x_{22}<\frac{\left(r-\varepsilon_{1}\right)}{r \eta} .
\end{aligned}
$$

Similarly, according to Lemma $6, E_{p}$ is a pseudoequilibrium for $x_{21}<V_{T}<x_{11}$ or $x_{12}<V_{T}<x_{22}$.

Having discussed the existence of the pseudoequilibria, we take the derivative of $Q_{s}$ with respect to $y$ to analyze the stability of the pseudoequilibria. Note that

$$
\begin{aligned}
\frac{d Q_{s}}{d y} & \\
= & \left(-\frac{\varepsilon_{2}}{\varepsilon_{1}} p y+\frac{\varepsilon_{2}}{\varepsilon_{1}} r\left(1-\eta V_{T}\right)+\frac{c V_{T}}{1+\omega V_{T}}-q V_{T}-\delta\right) \\
& -\frac{\varepsilon_{2}}{\varepsilon_{1}} p y .
\end{aligned}
$$

Then, we have $\left.\left(d Q_{s} / d y\right)\right|_{E_{0}}=\left(\varepsilon_{2} / \varepsilon_{1}\right) r\left(1-\eta V_{T}\right)+c V_{T} /(1+$ $\left.\omega V_{T}\right)-q V_{T}-\delta=\left(\varepsilon_{2} / \varepsilon_{1}\right) p y_{p}$ and $\left.\left(d Q_{s} / d y\right)\right|_{E_{p}}=-\left(\varepsilon_{2} / \varepsilon_{1}\right) p y_{p}$.

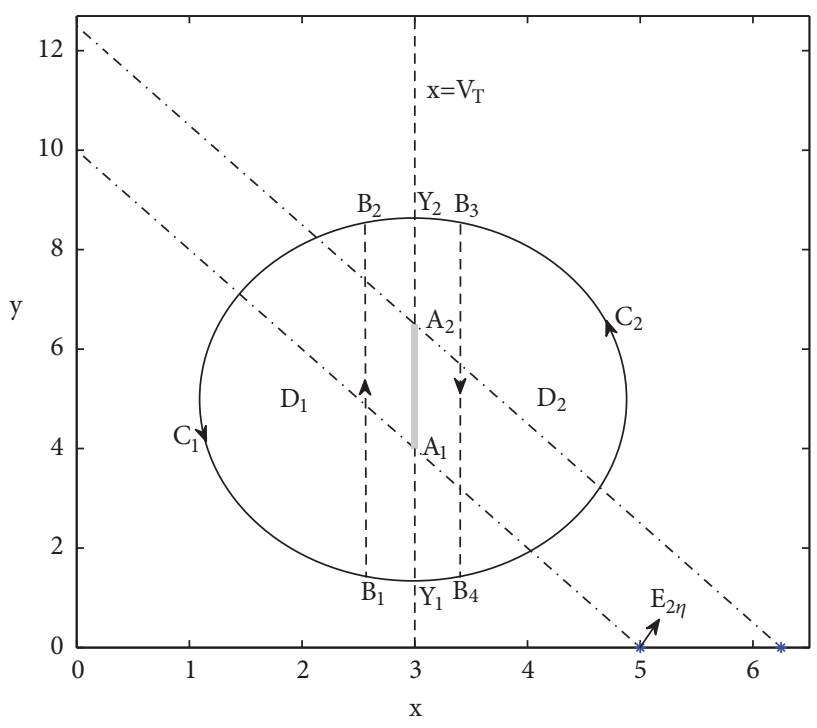

FIGURE 1: Phase plane $\mathrm{x}$-y for Filippov epidemic model (3), showing the switching line $\left(x=V_{T}\right)$, sliding segment $\overline{A_{1} A_{2}}$, and the diagrams $C_{1}$ and $C_{2}$ split from the limit cycle $\mathrm{C}$ by the switching line.

Therefore, if $\left(H_{1}\right)$ holds true, then the pseudoequilibrium $E_{p}$ is locally stable on the sliding segment when it is feasible, and $E_{0}$ is unstable; if $\left(\mathrm{H}_{2}\right)$ holds true, then $E_{p}$ becomes infeasible and the pseudoequilibrium $E_{0}$ is locally stable on the sliding segment when it exists.

\section{Global Dynamics of System (3)}

In this section, we will investigate the global dynamics of Filippov system (3). Initially, we shall rule out the existence of limit cycle for system (3). Here, we take Case A11 as an example to illustrate that there is not any limit cycles. The detailed proof is given in the appendix. Note that, for the other cases, we can prove the nonexistence of limit cycle for system (3) using the similar methods, and here we omit the detailed proof.

Lemma 8. For Case A11, there is no limit cycle for Filippov system (3).

Corresponding to the discussion of the sliding dynamics, we then consider the following cases to examine the global dynamics of system (3).

Case $A 1$. There is no positive equilibrium for subsystem $S_{2}$.

(i) There is no positive equilibrium for subsystem $S_{1}$ (i.e., Case A11). Then, we discuss the global dynamics of the Filippov system by considering the following subsituations:

(a) If $0<V_{T}<x_{2 \eta}$, then we know that $E_{2 \eta}$ is a real equilibrium which is stable while $E_{1 \eta}$ is a virtual equilibrium which is unstable. As we mentioned above, there is no limit cycle for system (3), and moreover there is not any other stable equilibrium, thus the local stability of $E_{2 \eta}$ indicates that $E_{2 \eta}$ is globally asymptotically stable (shown in Figure 2(a)). 


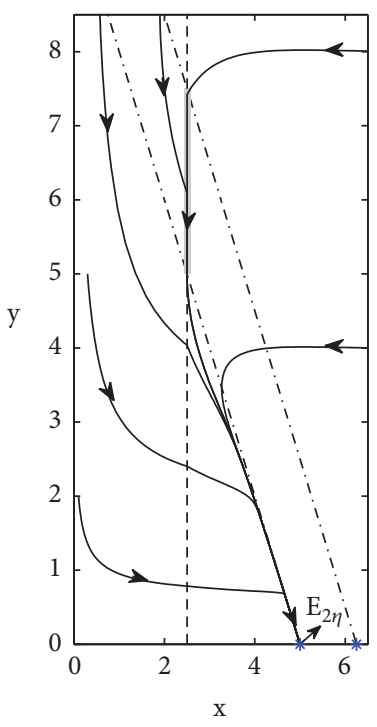

(a)

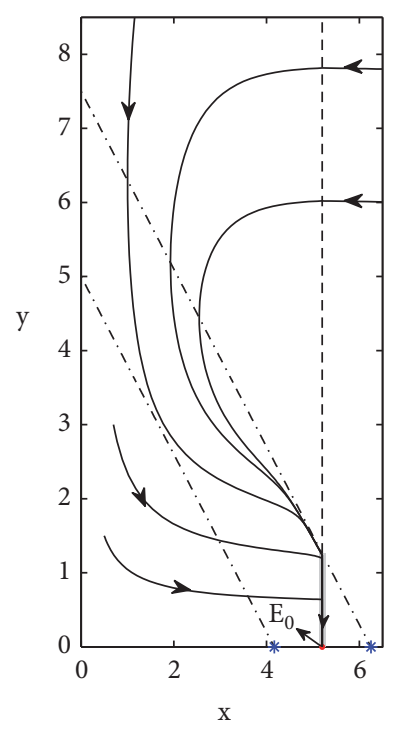

(b)

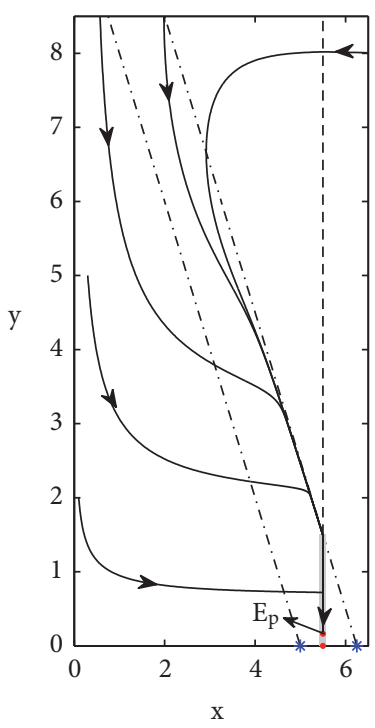

(c)

Figure 2: Phase portraits of Filippov system (3) for Case All with different threshold conditions. (a) The boundary equilibrium $E_{2 \eta}$ is globally asymptotically stable, here $V_{T}=2.5$; (b) the pseudoequilibrium $E_{0}$ is globally asymptotically stable, here $V_{T}=5.2$; (c) the pseudoequilibrium $E_{p}$ is globally asymptotically stable, here $V_{T}=5.5$. The other parameter values are fixed as $r=10, p=0.8, \omega=0.23, c=2, q=0.3, \delta=3.26$, $\eta=0.16, \varepsilon_{1}=2$, and $\varepsilon_{2}=0.1$.

(b) If $x_{2 \eta}<V_{T}<x_{1 \eta}$ and $\left(H_{2}\right)$ hold true, then we have that $E_{2 \eta}$ becomes a virtual equilibrium. It follows from the discussion in Section 3 that the pseudoequilibrium $E_{0}$ is stable on the sliding segment $\Sigma_{s}^{1}$. Similarly, system (3) does not have any limit cycle. Therefore, the local stability of $E_{0}$ indicates that $E_{0}$ is globally asymptotically stable (shown in Figure 2(b)).

(c) If $x_{2 \eta}<V_{T}<x_{1 \eta}$ and $\left(H_{1}\right)$ hold true, then there are two pseudoequilibria $E_{0}$ and $E_{p}$ on the sliding segment $\Sigma_{s}^{1}$. As we proved in Section $3, E_{p}$ is locally stable while $E_{0}$ is unstable. Similar to subcase (b), we have that $E_{p}$ is globally asymptotically stable (shown in Figure 2(c)).

Based on the above discussions, the global dynamics of system (3) can be concluded as follows.

Theorem 9. For Case A11, if $0<V_{T}<x_{2 \eta}$, then the boundary equilibrium $E_{2 \eta}$ is globally asymptotically stable; if $x_{2 \eta}<V_{T}<$ $x_{1 \eta}$, then the pseudoequilibrium $E_{0}$ is globally asymptotically stable for $\left(\mathrm{H}_{2}\right)$ being satisfied, while the pseudoequilibrium $E_{p}$ is globally asymptotically stable for $\left(H_{1}\right)$ being satisfied.

(ii) There is a unique positive equilibrium for subsystem $S_{1}$ (i.e., Case $A 12$ ). If $0<V_{T}<x_{2 \eta}, E_{1 \eta}$ and $E_{11}$ are virtual equilibria, and $E_{2 \eta}$ is a real equilibrium which is stable. As mentioned above, under this scenario, there is no limit cycle or no pseudoequilibrium for Filippov system (3), and thus $E_{2 \eta}$ is globally stable. If $x_{2 \eta}<V_{T}<x_{21}, E_{2 \eta}$ becomes a virtual equilibrium, and there emerges the stable pseudoequilibrium $E_{0}$ on the sliding segment $\Sigma_{s}^{1}$. If $x_{21}<V_{T}<x_{11}$, the same results hold compared with the case $x_{2 \eta}<V_{T}<x_{21}$ if $\left(H_{2}\right)$ holds true, while there are two pseudoequilibria $E_{0}$ (unstable) and $E_{p}$ (stable) on $\Sigma_{s}^{1}$ if $\left(H_{1}\right)$ holds true. Furthermore, If $x_{11}<$ $V_{T}<x_{1 \eta}, E_{11}$ becomes a real equilibrium which is locally stable. There is a pseudoequilibrium $E_{0}$ on $\Sigma_{s}^{1}$ which is unstable. Then, the global dynamics of Filippov system (3) are concluded as follows.

Theorem 10. For Case A12, if $0<V_{T}<x_{2 \eta}$, then $E_{2 \eta}$ is globally asymptotically stable (shown in Figure 3(a)); if $x_{2 \eta}<$ $V_{T}<x_{21}$, then $E_{0}$ is globally asymptotically stable (shown in Figure 3(b)); if $x_{21}<V_{T}<x_{11}$, then $E_{0}$ is globally asymptotically stable for $\left(\mathrm{H}_{2}\right)$ being satisfied and $E_{p}$ is globally asymptotically stable for $\left(H_{1}\right)$ being satisfied (shown in Figure 3(c)); if $x_{11}<V_{T}<x_{1 \eta}$, then $E_{11}$ is globally asymptotically stable (shown in Figure 3(d)).

(iii) There are two positive equilibria for subsystem $S_{1}$ (i.e., Case A13). If there is only one steady state (boundary equilibrium or positive equilibrium or pseudoequilibrium), then it is globally stable. We can analyze the dynamics of system (3) under the same scenario using the similar methods. However, there is a different situation for such case. That is, if $x_{12}<V_{T}<\min \left\{x_{1 \eta}, x_{22}\right\}$ holds true, then $E_{11}$ is a real equilibrium; hence it is locally stable. Furthermore, $E_{0}$ is a locally stable pseudoequilibrium if $\left(\mathrm{H}_{2}\right)$ holds true, but if $\left(H_{1}\right)$ holds true, $E_{0}$ lose its stability and $E_{p}$ becomes a locally stable pseudoequilibrium. In conclusion, if $x_{12}<$ $V_{T}<\min \left\{x_{1 \eta}, x_{22}\right\}, E_{11}$ is bistable with $E_{0}$ (or $E_{p}$ ) for $\left(H_{2}\right)$ (or $\left.\left(H_{1}\right)\right)$ being satisfied. Based on the discussion above, the global dynamics of system (3) for Case A13 can be concluded as follows.

Theorem 11. For Case A13, if $0<V_{T}<x_{2 \eta}$, then $E_{2 \eta}$ is globally asymptotically stable (shown in Figure 4(a)); if $x_{2 \eta}<$ $V_{T}<x_{21}$, then $E_{0}$ is globally asymptotically stable (shown in Figure 4(b)); if $x_{21}<V_{T}<x_{11}$, then $E_{0}$ (or $E_{p}$ ) is globally 


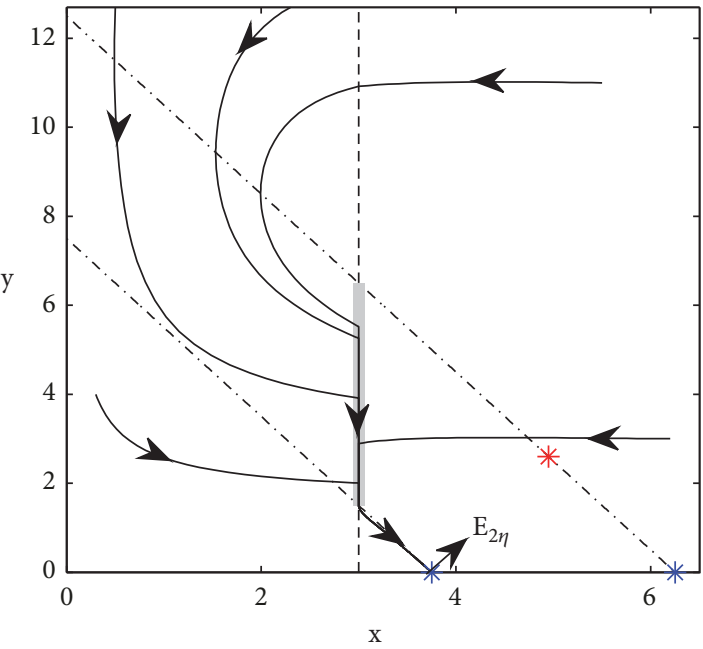

(a)

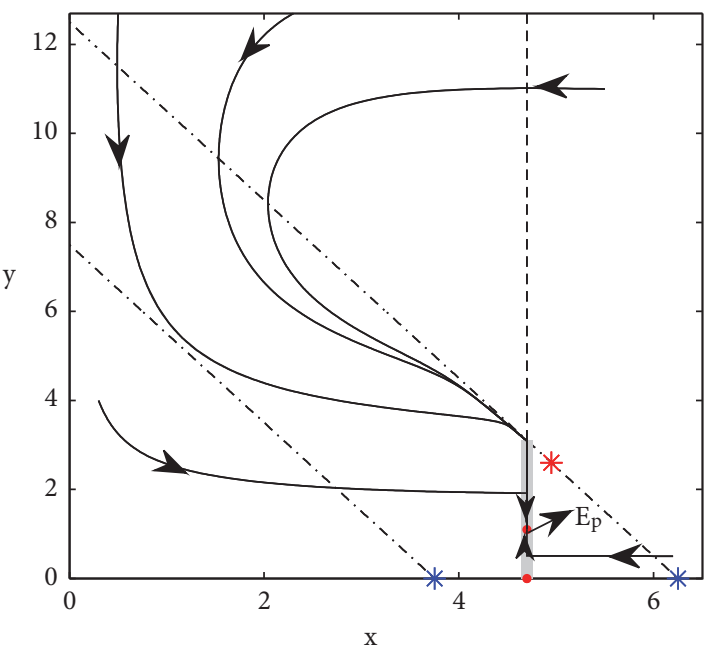

(c)

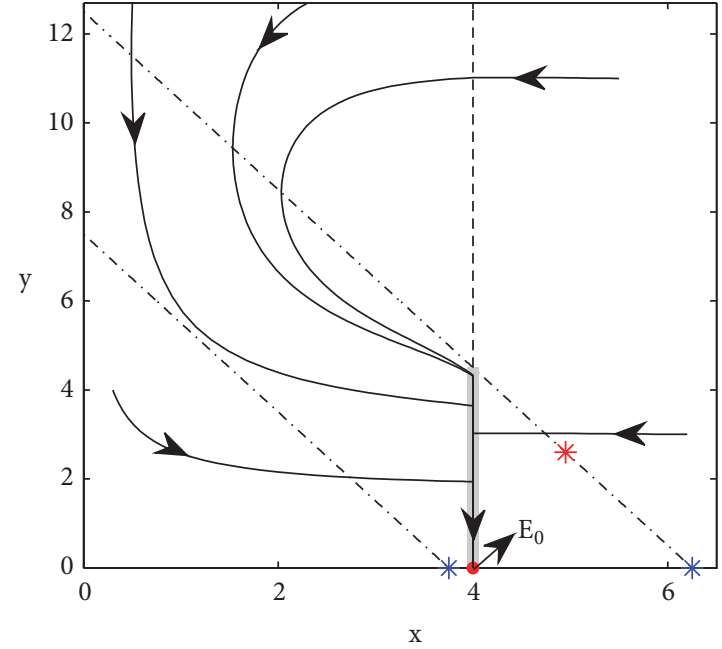

(b)

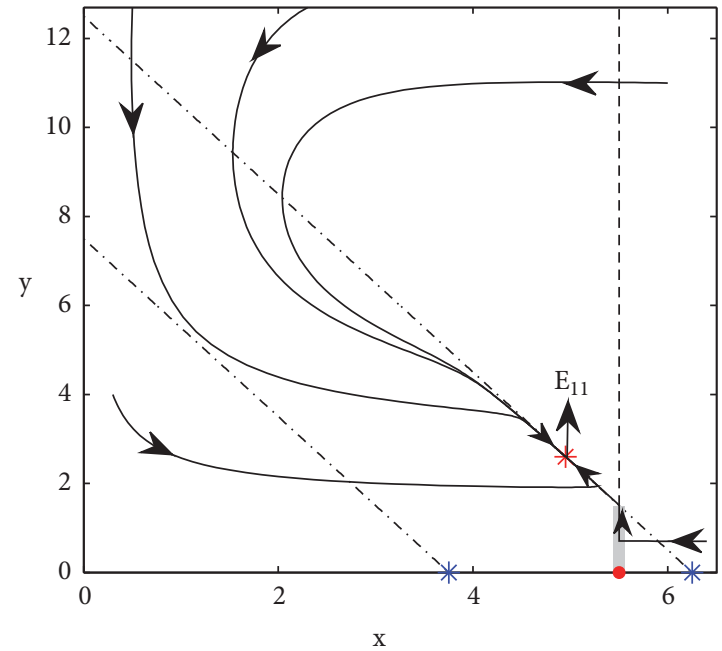

(d)

Figure 3: Phase plane of Filippov system (3) for Case $A 12$ with different threshold conditions. (a) The boundary equilibrium $E_{2 \eta}$ is globally asymptotically stable, here $V_{T}=3$; (b) the pseudoequilibrium $E_{0}$ is globally asymptotically stable, here $V_{T}=4$; (c) the pseudoequilibrium $E_{p}$ is globally asymptotically stable, here $V_{T}=4.7 ;$ (d) the regular equilibrium $E_{11}$ is globally asymptotically stable, here $V_{T}=5.5$. Parameter values are $r=10, p=0.8, \omega=0.23, c=2.05, q=0.3, \delta=3.26, \eta=0.16, \varepsilon_{1}=4$, and $\varepsilon_{2}=0.1$.

asymptotically stable for $\left(H_{2}\right)$ (or $\left(H_{1}\right)$ ) being satisfied (shown in Figure 4(c) (or Figure 4(d))); if $x_{11}<V_{T}<x_{12}$, then $E_{11}$ is globally asymptotically stable (shown in Figure 4(e)); if $x_{12}<$ $V_{T}<\min \left\{x_{1 \eta}, x_{22}\right\}$, then $E_{0}$ and $E_{11}$ are bistable for $\left(H_{2}\right)$ being satisfied and $E_{p}$ and $E_{11}$ are bistable for $\left(H_{1}\right)$ being satisfied (shown in Figure $4(f))$. In particular, $E_{0}$ and $E_{11}$ are always bistable for $x_{22}<V_{T}<x_{1 \eta}$.

Cases $A 2(A 21, A 22$, and $A 23)$ and $A 31$. All the dynamics of system (3) for four cases are similar to the former three cases, and thus we just omit the proof here. The global dynamics of these cases are summarized in Table 1 with the dynamical behaviors shown in Figures 5-8, respectively.

Case A32. There are two positive equilibria for both subsystems $S_{1}$ and $S_{2}$, respectively. And the horizontal compartments of these equilibria satisfy $x_{21}<x_{11}<x_{12}<x_{22}$. In terms of the existence and stability of the positive equilibria, the dynamics of the subsystems are similar to the focused situation in study [30]. Thus, based on the relationships of $V_{T}$ with respect to $x_{21}, x_{11}, x_{12}$, and $x_{22}$, we can analyze the dynamics of system (3) through the following subcases.

If the threshold condition is relatively small, i.e., $0<V_{T}<$ $x_{21}$, only $E_{2 \eta}, E_{21}$, and $E_{22}$ are real equilibria. It follows from the dynamics of system $S_{2}$ that both $E_{2 \eta}$ and $E_{21}$ are stable, and thus they are bistable for the Filippov system (3). If $V_{T}$ increases and satisfies $x_{21}<V_{T}<x_{11}, E_{21}$ becomes a virtual equilibrium while the locally stable pseudoequilibrium $E_{p}$ emerges, and thus $E_{2 \eta}$ and $E_{p}$ are bistable. Further, we can conclude that $E_{11}$ is bistable with $E_{2 \eta}$ when $x_{11}<V_{T}<x_{12}$ or $x_{22}<V_{T}<x_{2 \eta}$, while it is bistable with $E_{0}$ when $x_{2 \eta}<V_{T}<$ $x_{1 \eta}$. If $x_{12}<V_{T}<x_{22}, E_{11}$ and $E_{2 \eta}$ are stable while there also exists pseudoequilibrium $E_{p}$ which is locally stable as well. 


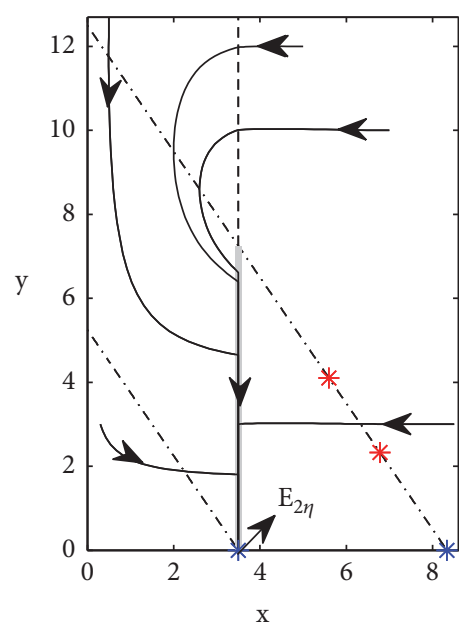

(a)

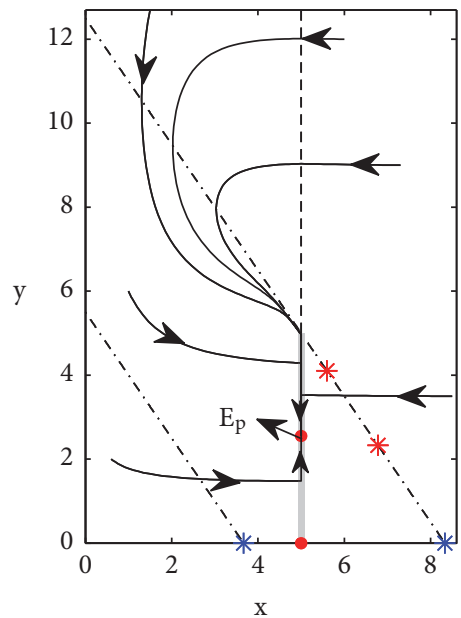

(d)

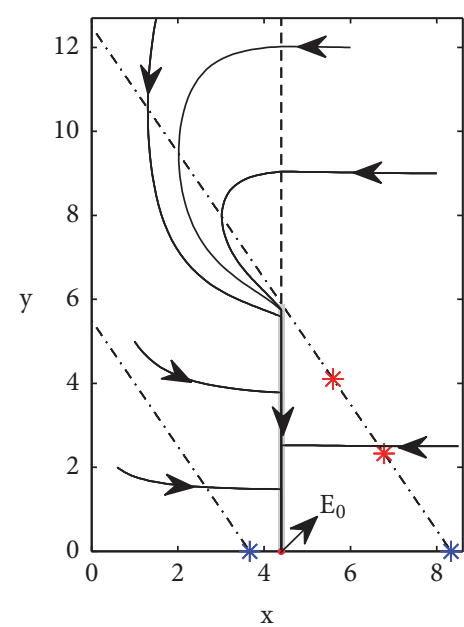

(b)

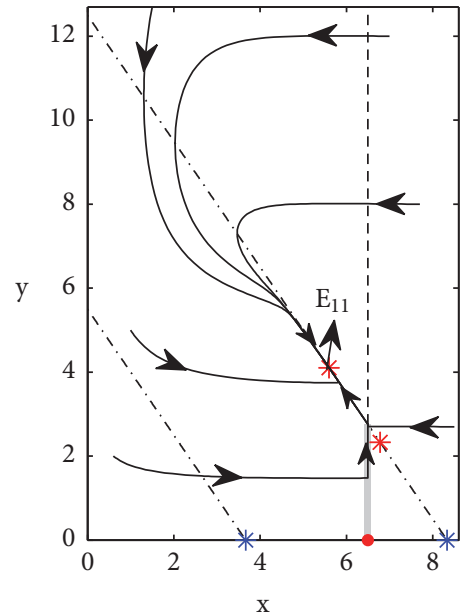

(e)

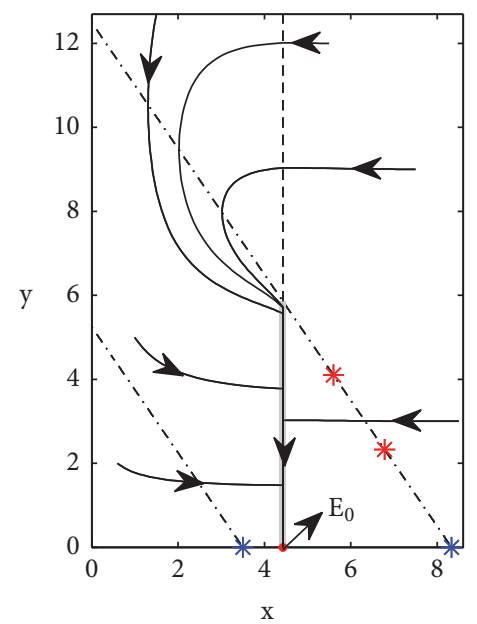

(c)

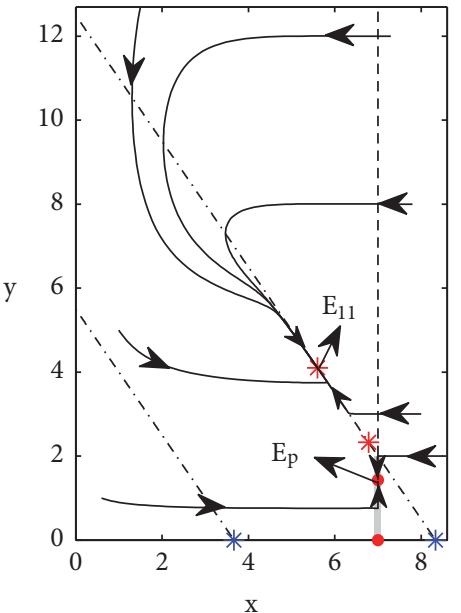

(f)

FIGURE 4: Solution trajectories of Filippov system (3) for Case $A 13$ with different threshold conditions. (a) The boundary equilibrium $E_{2 \eta}$ is globally asymptotically stable, here $V_{T}=3.5$; (b) the pseudoequilibrium $E_{0}$ is globally asymptotically stable, here $V_{T}=4.4$; (c) the pseudoequilibrium $E_{0}$ is globally asymptotically stable, here $V_{T}=4.43$; (d) the pseudoequilibrium $E_{p}$ is globally asymptotically stable, here $V_{T}=5$; (e) the regular equilibrium $E_{11}$ is globally asymptotically stable, here $V_{T}=6.5$; (f) the regular equilibrium $E_{11}$ and the pseudoequilibrium $E_{p}$ are bistable, here $V_{T}=7$. Parameter values are chosen as follows: $r=10, p=0.8, \omega=0.24, c=1.848, q=0.3$, $\delta=2.735, \eta=0.12, \varepsilon_{1}=5.6$, and $\varepsilon_{2}=0.1$.

Thus, based on the above discussions, we have the following conclusions.

Theorem 12. For Case A32, if $0<V_{T}<x_{21}$, then equilibrium $E_{21}$ and the boundary equilibrium $E_{2 \eta}$ are bistable (shown in Figure 9(a)); if $x_{21}<V_{T}<x_{11}$, then $E_{p}$ and $E_{2 \eta}$ are bistable (shown in Figure 9(b)); if $x_{11}<V_{T}<x_{12}$, then $E_{11}$ and $E_{2 \eta}$ are bistable (shown in Figure 9(c)); if $x_{12}<V_{T}<x_{22}$, then $E_{p}, E_{11}$, and $E_{2 \eta}$ are locally tristable (shown in Figure 9(d)); if $x_{22}<$ $V_{T}<x_{2 \eta}, E_{2 \eta}$ and $E_{11}$ are bistable (shown in Figure 9(e)); if $x_{2 \eta}<V_{T}<x_{1 \eta}$, then $E_{0}$ and $E_{11}$ are bistable (shown in Figure $9(f)$ ).

\section{Conclusion and Discussion}

Based on the basic immune-virus model, we considered the threshold policy depending on virus loads for the
HIV-infected patients and obtained a nonsmooth system. Compared with the model in [30], we extend it by taking the saturated growth of virus into consideration, and hence our model is better in line with the biological reality. Meanwhile, our model also includes a piecewise elimination rate of HIV virus and growth rate of the effector cells to indicate that the antiretroviral therapy and interleukin-2 (IL-2) treatment are carried out simultaneously once the HIV virus loads increase above a threshold level.

We first briefly concluded the dynamics of two subsystems. Based on the properties of the subsystems, we discussed the sliding mode and sliding dynamics with different threshold conditions. In more detail, the sliding segments are $\Sigma_{s}^{1}=\left\{(x, y) \mid x=V_{T}, 0 \leq y \leq(r / p)\left(1-\eta V_{T}\right)\right\}$, for $x_{2 \eta}<V_{T}<x_{1 \eta}$, and $\Sigma_{s}^{2}=\left\{x, y \mid x=V_{T},(r / p)\left(1-\eta V_{T}\right)-\right.$ $\left.\varepsilon_{1} / p \leq y \leq(r / p)\left(1-\eta V_{T}\right)\right\}$ for $0<V_{T}<x_{2 \eta}$. We note that 


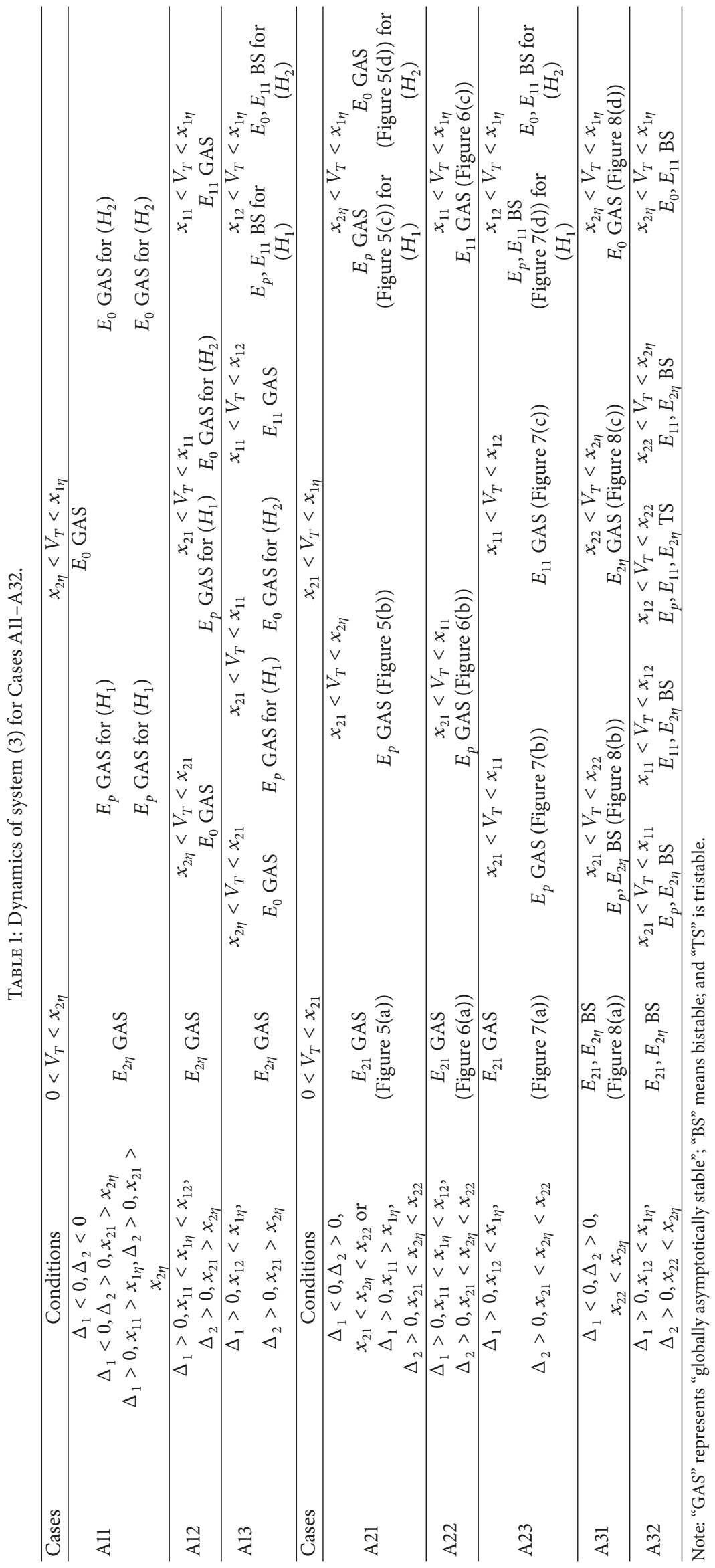




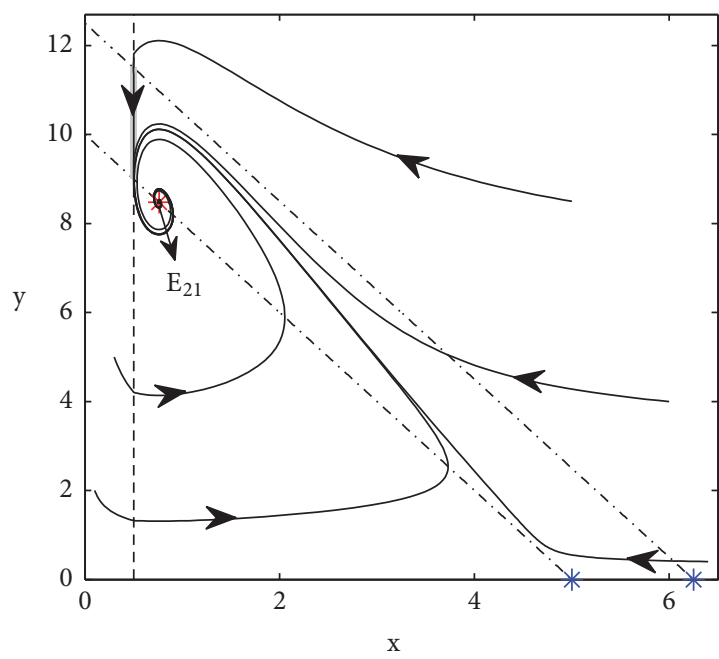

(a)

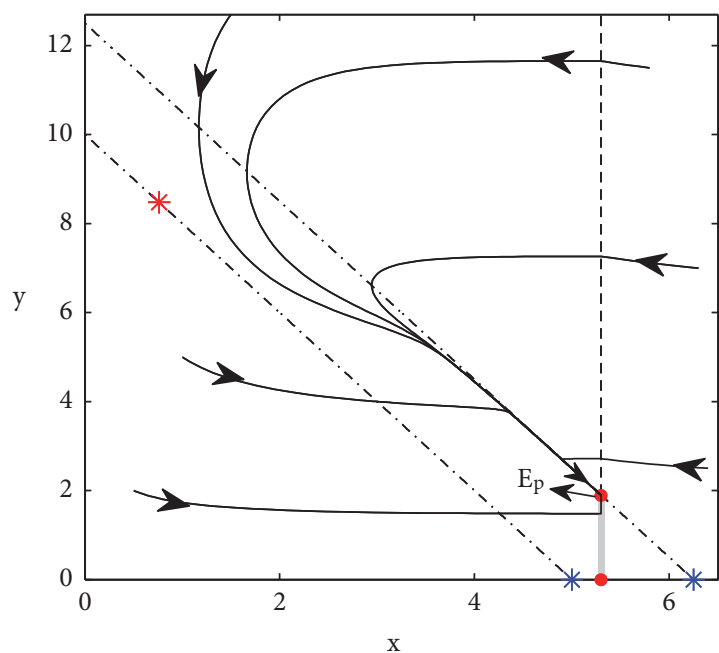

(c)

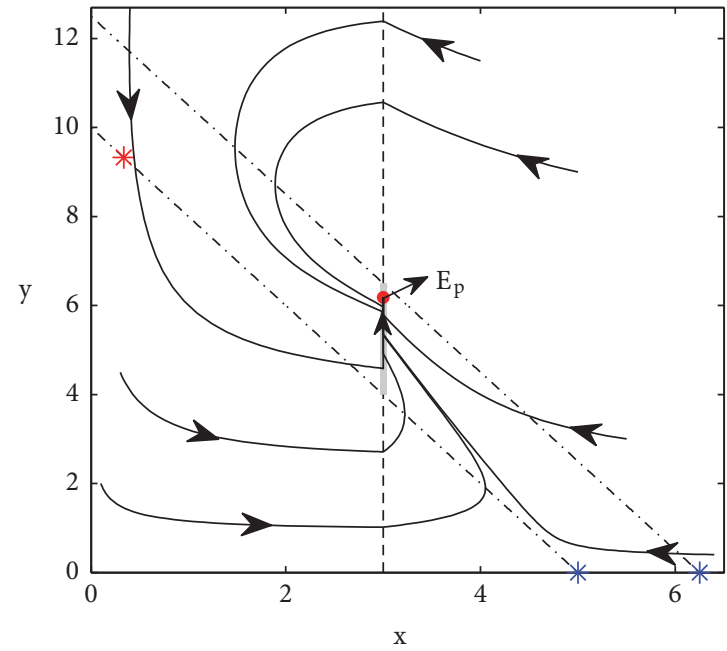

(b)

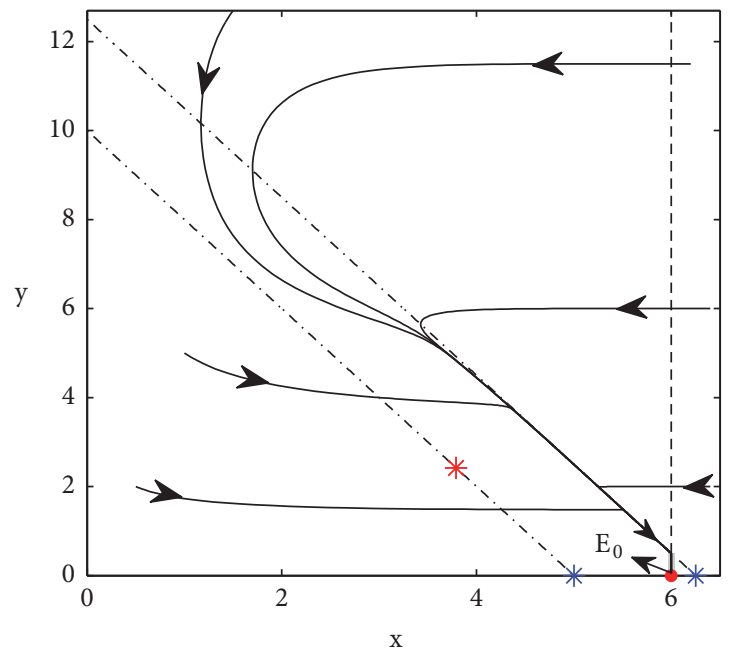

(d)

Figure 5: Phase portraits of Filippov system (3) for Case $A 21$ with different threshold conditions. (a) the regular equilibrium $E_{21}$ is globally asymptotically stable, here $V_{T}=1.5 ; \varepsilon_{2}=1.5$; (b) the pseudoequilibrium $E_{p}$ is globally asymptotically stable, here $V_{T}=3 ; \varepsilon_{2}=2 ;(\mathrm{c})$ the pseudoequilibrium $E_{p}$ is globally asymptotically stable, here $V_{T}=5.3 ; \varepsilon_{2}=1.5 ;$ (d) the pseudoequilibrium $E_{0}$ is globally asymptotically stable, here $V_{T}=6 ; \varepsilon_{2}=0.1$. All the other parameter values are $r=10, p=0.8, \omega=0.3, c=2, q=0.3, \delta=2.51, \eta=0.16$, and $\varepsilon_{1}=2$.

there is always a pseudoequilibrium $E_{0}$ on the sliding segment $\Sigma_{s}^{1}$. And we further discussed the existence of the other pseudoequilibrium $E_{p}$ by considering eight different cases. Correspondingly, we then investigated the global dynamics of system (3) for various cases. Our main results show that the system can exhibit very rich dynamic behaviors: (a) one of the equilibria is globally asymptotically stable, which can be the pseudoequilibrium $E_{0}$ or the pseudoequilibrium $E_{p}$ or the boundary equilibrium $E_{2 \eta}$ of subsystem $S_{2}$ or the positive equilibrium $E_{11}$ or $E_{21}$ of subsystem $S_{1}$ or $S_{2}$; (b) two equilibria are bistable, particularly, which can be that the positive equilibrium $E_{11}$ is bistable with $E_{0}$ (or $E_{p}$ or $\left.E_{2 \eta}\right)$, or the boundary equilibrium $E_{2 \eta}$ is bistable with the pseudoequilibrium $E_{p}$ (or the positive equilibrium $E_{21}$ ); (c) three equilibria are locally tristable; that is, the pseudoequilibrium $E_{p}$, the boundary equilibrium $E_{2 \eta}$, and the positive equilibrium $E_{11}$ are stable for $x_{12}<V_{T}<x_{22}$.
Global dynamics of our proposed model means that there is the case that the boundary equilibrium is globally stable for both the subsystems. Note that the immune-virus system without any therapy is actually system $S_{1}$ (the free system) while the immune-virus system with continuous therapy can be described by system $S_{2}$ (the control system). Comparing system $S_{2}$ with system $S_{1}$, we can find that the continuous therapy can shrink the final density of the virus (i.e., $x_{2 \eta}<$ $\left.x_{1 \eta}\right)$. It follows that the continuous therapy failed to maintain the activity of the immune system since the effector cell goes to zero finally. However, threshold policy indicated that, with a proper threshold condition, the STIs can not only successfully control virus loads to a previously given level which can be far below its natural carrying capacity (i.e., $x_{1 \eta}$ ) but also maintain the activity of the immune system (corresponding to the case that the pseudoequilibrium $E_{p}$ is globally stable), as Theorem 9 suggested. 


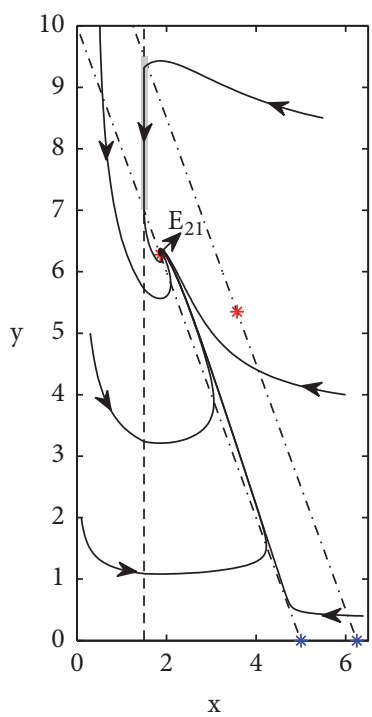

(a)

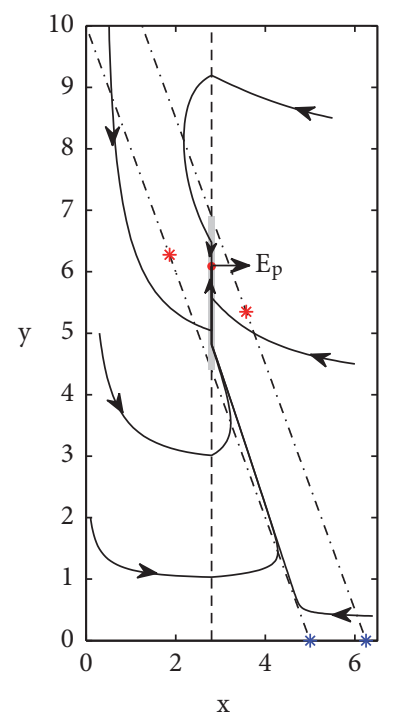

(b)

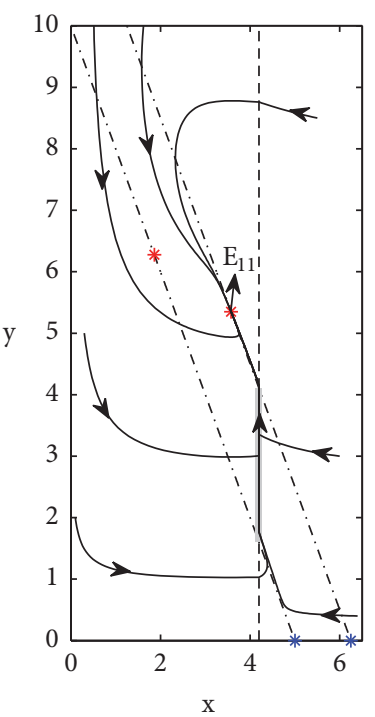

(c)

FIGURE 6: Phase portraits of Filippov system (3) for Case $A 22$ with different threshold conditions. (a) The regular equilibrium $E_{21}$ is globally asymptotically stable, here $V_{T}=1.5$; (b) the pseudoequilibrium $E_{p}$ is globally asymptotically stable, here $V_{T}=2.8$; (c) the regular equilibrium $E_{11}$ is globally asymptotically stable, here $V_{T}=4.2$. Parameter values are $c=2.1, \delta=2.55$, and $\varepsilon_{2}=0.6$, and other parameters are chosen as shown in Figure 5.

Moreover, according to the dynamics of subsystem $S_{1}$ for Case $A 21$ we can see that virus loads will finally approach its carrying capacity if there is no therapy for the patients. With the continuous therapy, the virus loads of the patients can be successfully controlled far below its carrying capacity and their immune system can always maintain the activity; that is, equilibrium $E_{21}$ is globally asymptotically stable for system $S_{2}$. For the virus-guided threshold policy, if the threshold level is set to satisfy $x_{21}<V_{T}<x_{2 \eta}$, then the pseudoequilibrium $E_{p}$ is globally stable, and $E_{p}$ will tend to $E_{21}$ as $V_{T} \longrightarrow x_{21}$. This means that the threshold policy can obtain the similar effects for the patients compared with the continuous therapy in terms of virus loads being controlled, which is in agreement with the clinical study [7].

It is worth mentioning that if we also assume that the two subsystems have two positive equilibria (i.e., Case A32), our model results, due to the saturated growth in model equations, show that the virus can not grow to infinity. Under this situation, we also found that the dynamics of system (3) are similar to those of the control system $\left(\right.$ system $S_{2}$ ) when the threshold condition is relatively small (i.e., $0<V_{T}<x_{21}$ ); that is, the positive equilibrium $E_{21}$ and the boundary equilibrium $E_{2 \eta}$ are bistable. And if the threshold is relatively high (i.e., $V_{T}>x_{22}$ ), the dynamics of system (3) are similar to the free system $S_{1}$. Moreover, for middle values of the threshold (i.e., $x_{12}<V_{T}<x_{22}$ ), then equilibria $E_{p}, E_{11}$, and $E_{2 \eta}$ are locally tristable. Therefore, the bistability and the tristability suggest that the optimal strategy should also be individual-based by taking the initial conditions of the patients and the threshold conditions into consideration.

\section{Appendix}

\section{Proof of Lemma 8}

As mentioned in Section 2, we have precluded the existence of limit cycle for system $S_{1}$ or system $S_{2}$. Then, we will first prove that no limit cycle exists containing part of the sliding segment $\overline{A_{1} A_{2}}$. Thus, we consider the following situations:

(a) If $0<V_{T}<x_{2 \eta}$, there is no pseudoequilibrium and the sliding dynamic equation (25) satisfies $d y / d t<0$, so the trajectory on the sliding segment $\overline{A_{1} A_{2}}$ moves from the top to the bottom. Therefore, in the region $x>V_{T}$ in Figure 1, we need to show that any orbit starting from $A_{1}$ cannot hit $\overline{A_{1} A_{2}}$ again; if it does not hold, it contradicts the stability of $E_{2 \eta}$ of the subsystem $S_{2}$. Therefore, no closed orbit containing part of the sliding segment $\overline{A_{1} A_{2}}$ exists.

(b) If $x_{2 \eta}<V_{T}<x_{1 \eta}$ and $\left(H_{1}\right)$, we have that $E_{p}$ is locally asymptotically stable on the sliding segment $\Sigma_{c}^{1}$. The local stability of $E_{p}$ implies that there is no limit cycle containing part of the sliding segment $\overline{A_{1} A_{2}}$.

(c) If $x_{2 \eta}<V_{T}<x_{1 \eta}$ and $\left(H_{2}\right)$ hold true, we have that $E_{0}$ is locally asymptotically stable on the sliding segment $\Sigma_{c}^{1}$. The local stability of $E_{0}$ on the sliding segment implies that there is no limit cycle containing part of the sliding segment $\overline{A_{1} A_{2}}$.

Next, we preclude the limit cycle for Filippov system (3) which surrounds the sliding segment $\overline{A_{1} A_{2}}$. Denote the right-hand side of (3) in the region $x<V_{T}$ (or $x>V_{T}$ ) by $f^{1}(z)$ (or $\left.f^{2}(z)\right)$, where $f^{i}(z)=\left(f^{i 1}(z), f^{i 2}(z)\right)$. We consider that $\mathrm{C}$ is a limit cycle surrounding the sliding segment $\overline{A_{1} A_{2}}$. Let $C_{1}\left(C_{2}\right)$ be its part on the left (right) side of the line $x=V_{T}$. 


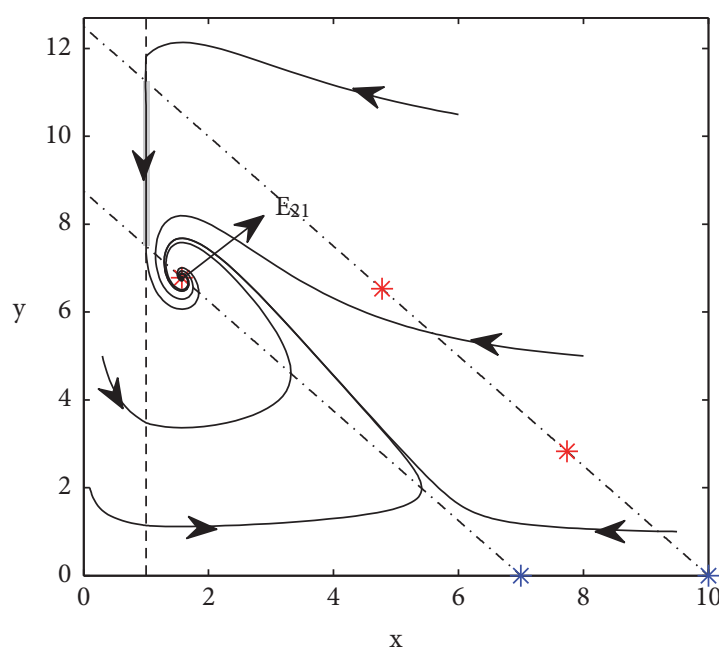

(a)

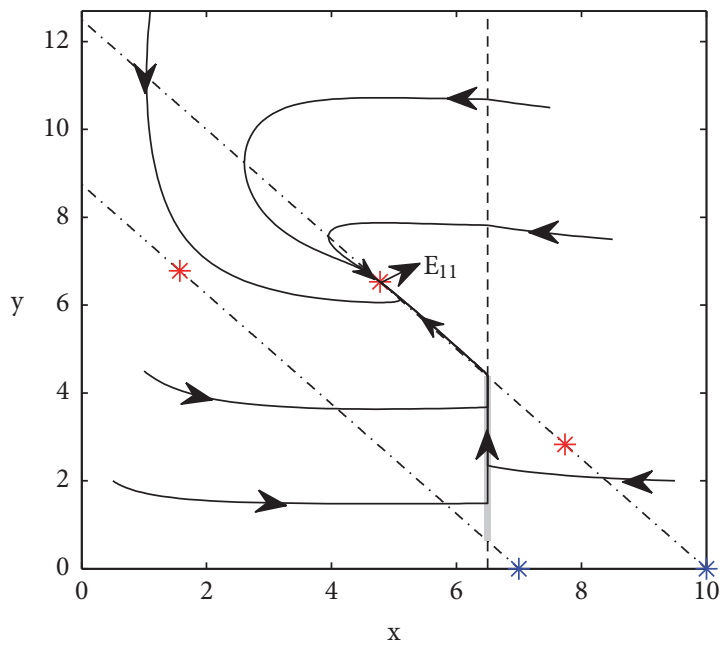

(c)

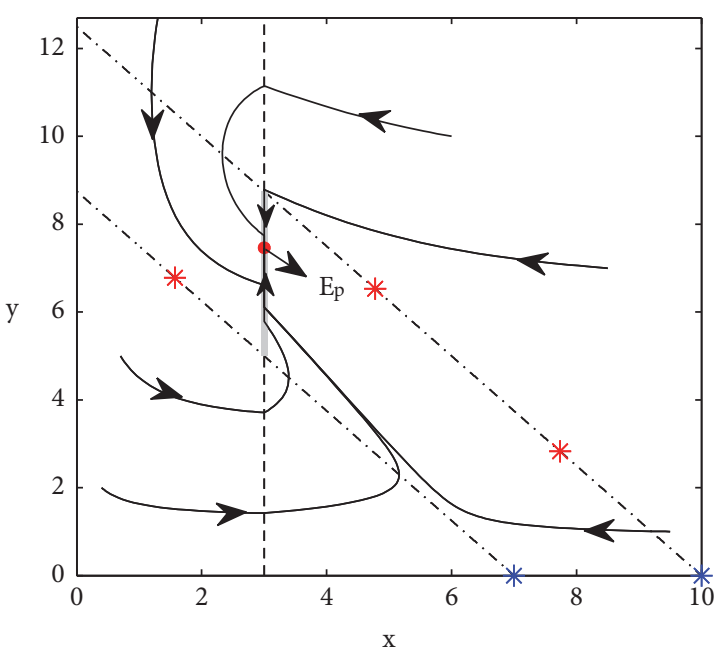

(b)

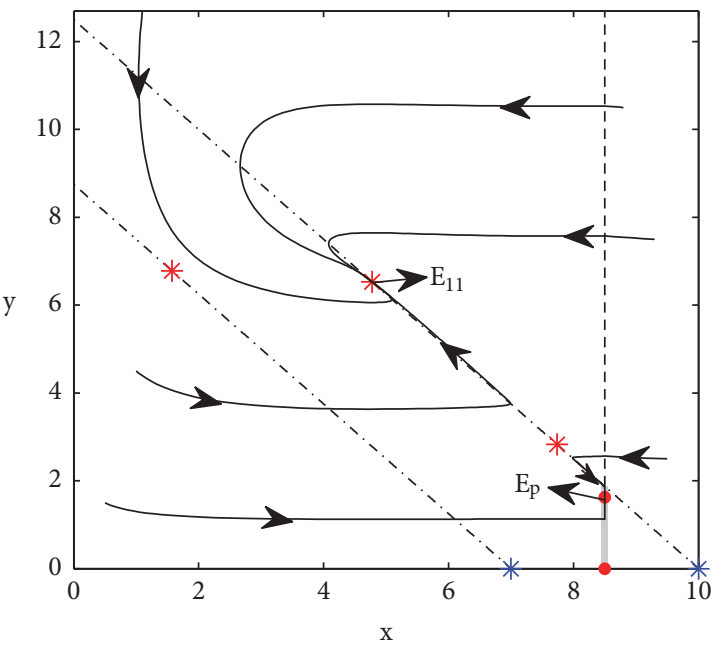

(d)

Figure 7: Solution trajectories of Filippov system (3) for Case $A 23$ with different threshold conditions. (a) the regular equilibrium $E_{21}$ is globally asymptotically stable, here $V_{T}=1$; (b) the pseudoequilibrium $E_{p}$ is globally asymptotically stable, here $V_{T}=3$; (c) the regular equilibrium $E_{11}$ is globally asymptotically stable, here $V_{T}=6.5$; (d) the regular equilibrium $E_{11}$ and the pseudoequilibrium $E_{p}$ are bistable, here $V_{T}=8.5$. Here $r=10, p=0.8, \omega=0.23, c=1.75, q=0.3, \delta=2.55, \eta=0.1, \varepsilon_{1}=3$, and $\varepsilon_{2}=1$.

Denoting that the limit cycle $\mathrm{C}$ and the line $x=V_{T}$ intersect at $Y_{1}$ and $Y_{2}$, the intersection points of $C$ and the two auxiliary lines $x=V_{T}-\varepsilon$ and $x=V_{T}+\varepsilon$ by $B_{1}, B_{2}, B_{3}, B_{4}$, respectively, are shown in Figure 1, where $\varepsilon(\varepsilon>0)$ is any sufficiently small number. Denote the region delimited by $C_{1}\left(C_{2}\right)$ and the segment $\overline{B_{1} B_{2}}\left(\overline{B_{3} B_{4}}\right)$ by $D_{1}\left(D_{2}\right)$, and the boundaries of $D_{1}$ and $D_{2}$ are denoted by $E_{1}$ and $E_{2}$. Define the Dulac function by $K=1 / x y$ as before. From the discussions given above and Green's theorem, we have

$$
\begin{aligned}
& \iint_{D_{1}}\left(\frac{\alpha\left(K f^{11}\right)}{\alpha x}+\frac{\alpha\left(K f^{12}\right)}{\alpha y}\right) d x d y \\
& \quad=\oint_{E_{1}} K\left(f^{11} d y-f^{12} d x\right)=\int_{\overrightarrow{B_{1} B_{2}}} K f^{11} d y,
\end{aligned}
$$

$$
\begin{aligned}
& \iint_{D_{2}}\left(\frac{\alpha\left(K f^{21}\right)}{\alpha x}+\frac{\alpha\left(K f^{22}\right)}{\alpha y}\right) d x d y \\
& \quad=\oint_{E_{2}} K\left(f^{21} d y-f^{22} d x\right)=\int_{\overrightarrow{B_{3} B_{4}}} K f^{21} d y .
\end{aligned}
$$

Let $D_{0} \subset D_{2}$ and 


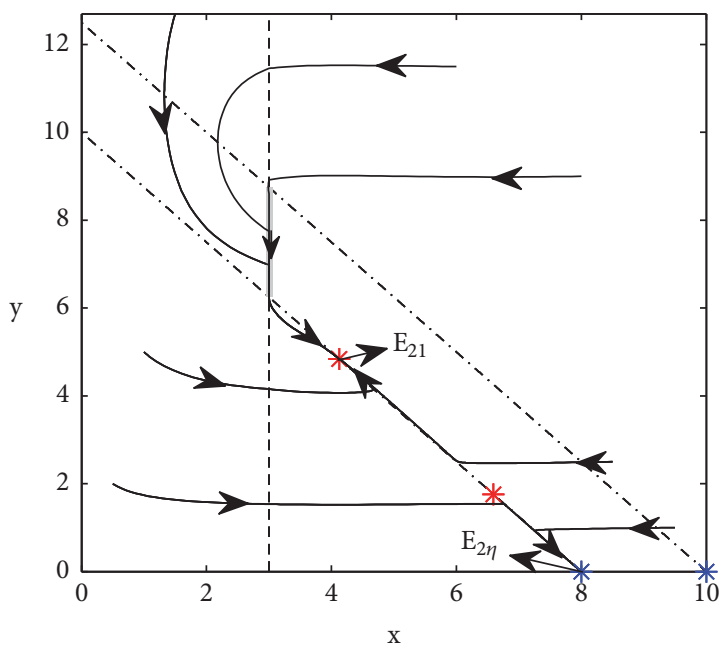

(a)

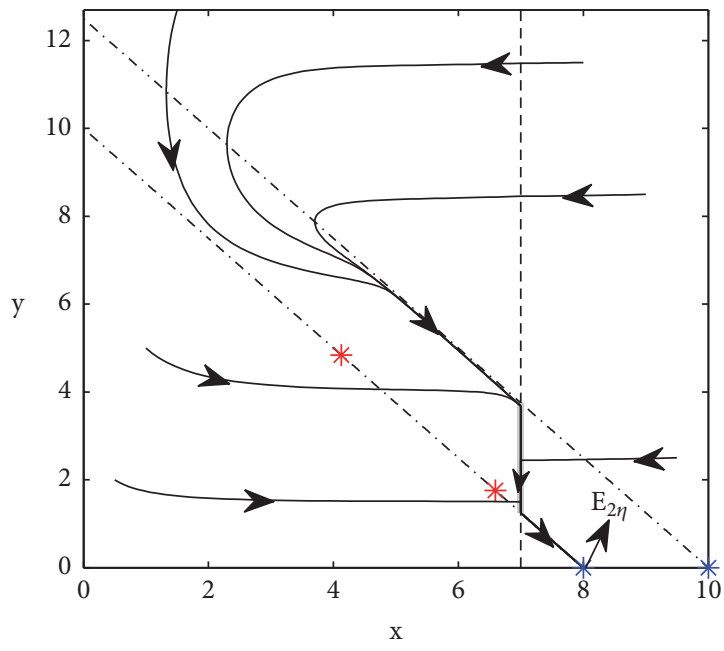

(c)

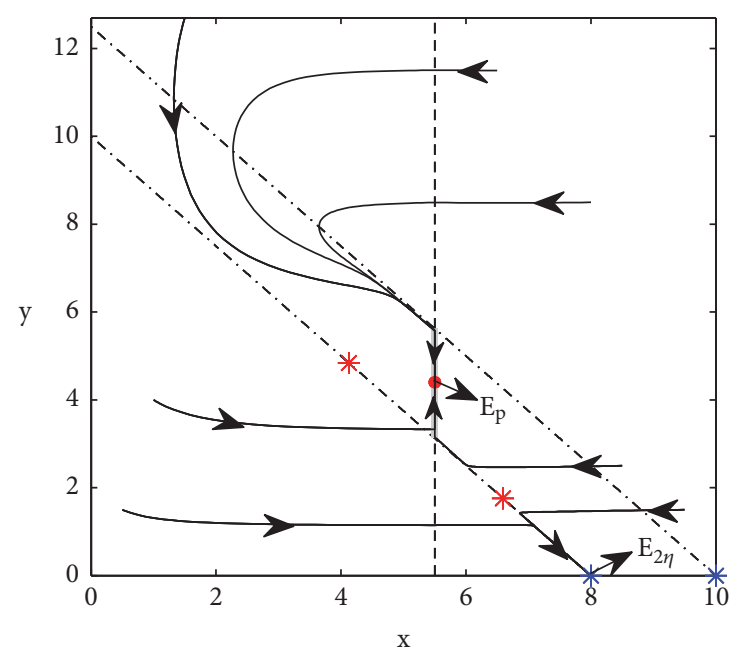

(b)

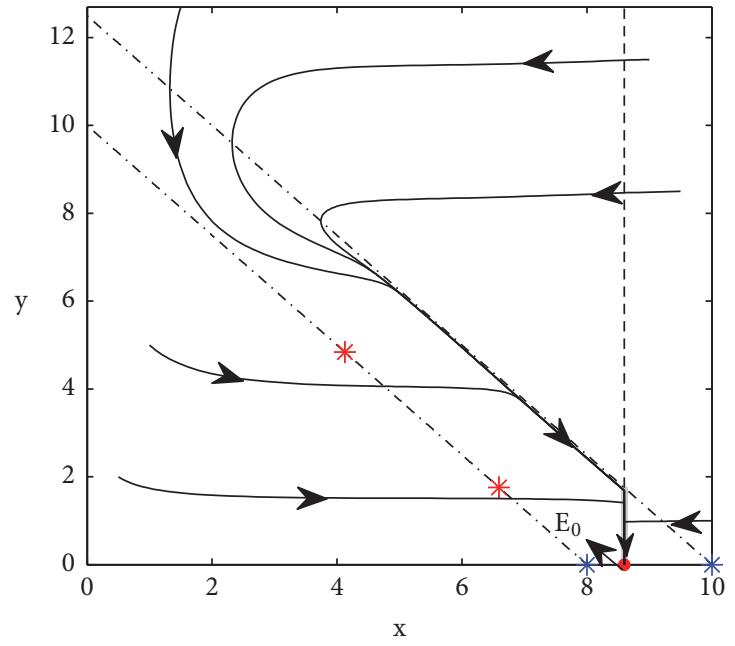

(d)

Figure 8: Phase plane of Filippov system (3) for Case $A 31$ with different threshold conditions. (a) The regular equilibrium $E_{21}$ and the boundary equilibrium $E_{2 \eta}$ are bistable, here $V_{T}=3$; (b) the pseudoequilibrium $E_{p}$ and the boundary equilibrium $E_{2 \eta}$ are bistable, here $V_{T}=$ 5.5; (c) the boundary equilibrium $E_{2 \eta}$ is globally asymptotically stable, here $V_{T}=7$; (d) the pseudoequilibrium $E_{0}$ is globally asymptotically stable. Parameter values are chosen as follows: $\delta=2.55, \eta=0.1$, and $\varepsilon_{2}=0.1$, and other parameter values are shown in Figure 5 .

since $\gamma<0$, and, furthermore,

$$
\begin{aligned}
& \iint_{D_{1}}\left(\frac{\alpha\left(K f^{11}\right)}{\alpha x}+\frac{\alpha\left(K f^{12}\right)}{\alpha y}\right) d x d y \\
& \quad+\iint_{D_{2}}\left(\frac{\alpha\left(K f^{21}\right)}{\alpha x}+\frac{\alpha\left(K f^{22}\right)}{\alpha y}\right) d x d y<\gamma<0,
\end{aligned}
$$

and we get

$$
\int_{\overrightarrow{B_{1} B_{2}}} K f^{11} d y+\int_{\overrightarrow{B_{3} B_{4}}} K f^{21} d y<\gamma<0 .
$$

Suppose that the ordinates of the points $Y_{1}, Y_{2}, B_{1}, B_{2}, B_{3}, B_{4}$ are $\underline{x}, \bar{x}, \underline{x}+f_{1}(\varepsilon), \bar{x}-f_{2}(\varepsilon), \bar{x}-f_{3}(\varepsilon), \underline{x}+f_{4}(\varepsilon)$, where $f_{i}(\varepsilon)$ is continuous and satisfies $\lim _{\varepsilon \rightarrow 0} f_{i}(\varepsilon)=0, f_{i}(0)=0$. Thus, we get

$$
\begin{aligned}
& \lim _{\varepsilon \longrightarrow 0}\left[\int_{B_{1} B_{2}} K f^{11} d y\right] \\
& \quad=\lim _{\varepsilon \longrightarrow 0} \int_{\underline{x}+f_{1}(\varepsilon)}^{\bar{x}-f_{2}(\varepsilon)}\left[\frac{r}{y}(1-\eta x)-p\right] d y \\
& \quad=r\left(1-\eta V_{T}\right) \ln \frac{\bar{x}}{\underline{x}}-p(\bar{x}-\underline{x}) .
\end{aligned}
$$

Similarly, we have

$$
\lim _{\varepsilon \rightarrow 0}\left[\int_{\overrightarrow{B_{3} B_{4}}} K f^{12} d y\right]
$$




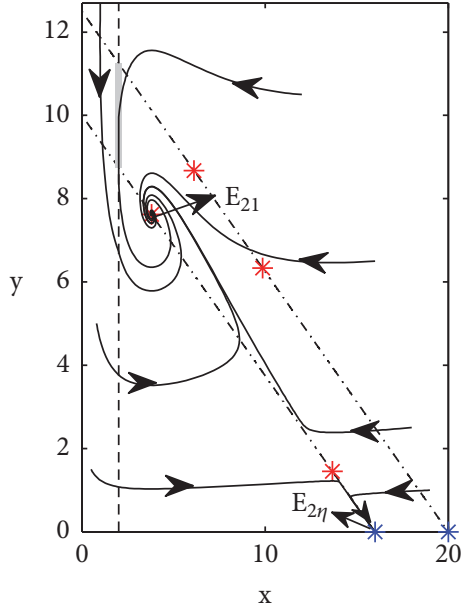

(a)

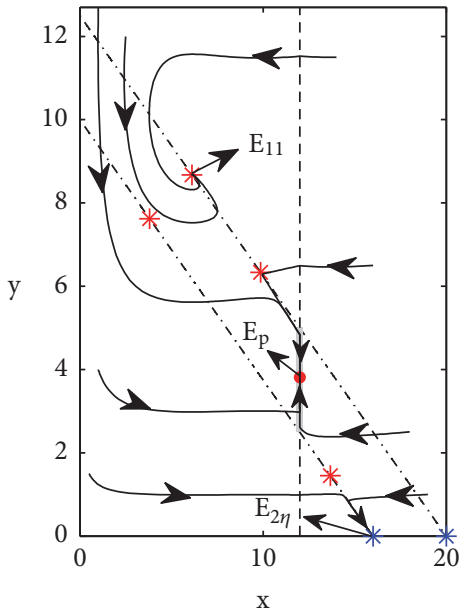

(d)

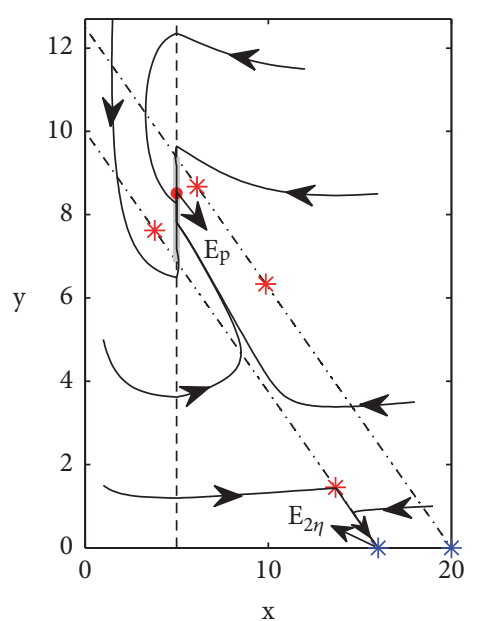

(b)

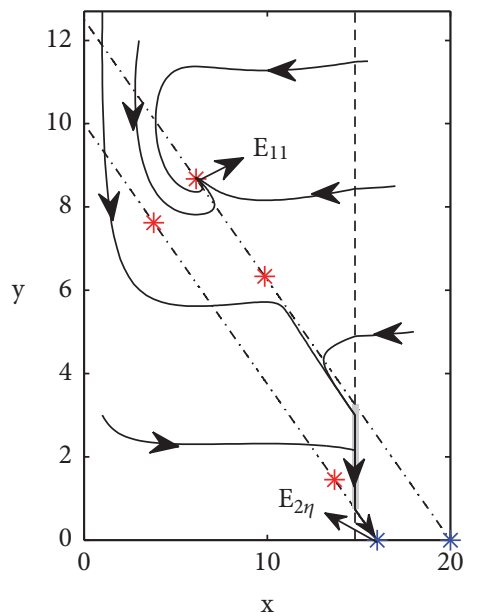

(e)

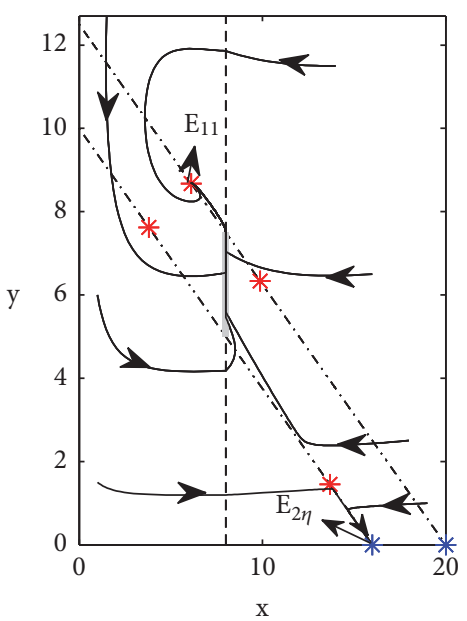

(c)

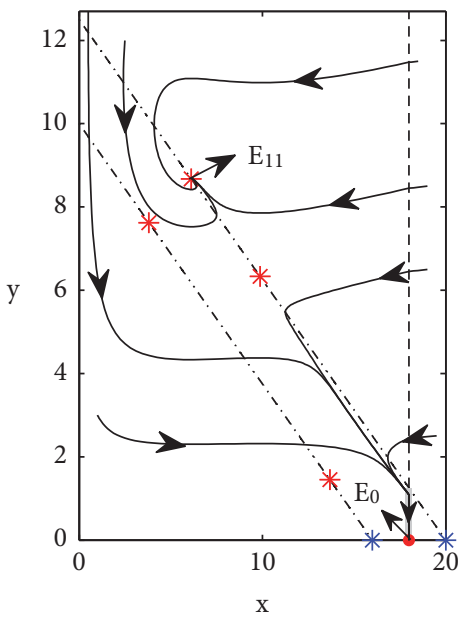

(f)

Figure 9: Phase plane of Filippov system (3) for Case $A 32$ with different threshold conditions. (a) The regular equilibrium $E_{21}$ and the boundary equilibrium $E_{2 \eta}$ are bistable, here $V_{T}=2$; (b) the pseudoequilibrium $E_{p}$ and the boundary equilibrium $E_{2 \eta}$ are bistable, here $V_{T}=5$; (c) the regular equilibrium $E_{11}$ and the boundary equilibrium $E_{2 \eta}$ are bistable, here $V_{T}=8$; (d) the regular equilibrium $E_{11}$, the pseudoequilibrium $E_{p}$ and the boundary equilibrium $E_{2 \eta}$ are locally stable simultaneously, here $V_{T}=12$; (e) the regular equilibrium $E_{11}$ and the boundary equilibrium $E_{2 \eta}$ are bistable, here $V_{T}=14.8$; (f) the regular equilibrium $E_{11}$ and the pseudoequilibrium $E_{0}$ are bistable, here $V_{T}=18$. We fix all other parameters as follows: $r=10, p=0.8, \omega=0.18, c=1.75, q=0.3, \delta=3.26, \eta=0.05, \varepsilon_{1}=2$, and $\varepsilon_{2}=0.45$.

$$
\begin{aligned}
& =\lim _{\varepsilon \rightarrow 0} \int_{\bar{x}-f_{3}(\varepsilon)}^{\underline{x}^{+} f_{4}(\varepsilon)}\left[\frac{r}{y}(1-\eta x)-p-\frac{\delta}{x}\right] d y \\
& =-r\left(1-\eta V_{T}\right) \ln \frac{\bar{x}}{\underline{x}}+\left(p+\frac{\delta}{V_{T}}\right)(\bar{x}-\underline{x}) .
\end{aligned}
$$

Therefore,

$$
\begin{aligned}
\lim _{\varepsilon \longrightarrow 0} & {\left[\int_{\overrightarrow{B_{1} B_{2}}} K f^{11} d y+\int_{\overrightarrow{B_{3} B_{4}}} K f^{21} d y\right]=\frac{\delta}{V_{T}}(\bar{x}-\underline{x}) } \\
& >0
\end{aligned}
$$

which contradicts (A.4). Thus, we preclude the existence of the limit cycle $C$ surrounding the sliding segment $\overline{A_{1} A_{2}}$. The proof is completed.

\section{Data Availability}

No data were used to support this study.

\section{Conflicts of Interest}

The authors declare that they have no conflicts of interest.

\section{Acknowledgments}

This project was partially supported by the National Natural Science Foundation of China (NSFC, Grant nos. 11631012 (Yanni Xiao) and 11571273 (Yanni Xiao)) and by the National Mega-project of Science Research (Grant no. 2017ZX10201101- 002-002 (Yanni Xiao)). The authors thank 
Professor Robert Cheke for his valuable comments and for his generous help in polishing the manuscript.

\section{References}

[1] F. J. Palella Jr., K. M. Delaney, A. C. Moorman et al., "Declining morbidity and mortality among patients with advanced human immunodeficiency virus infection," The New England Journal of Medicine, vol. 338, no. 13, pp. 853-860, 1998.

[2] A. Mocroft, S. Vella, T. L. Benfield et al., "Changing patterns of mortality across Europe in patients infected with HIV-1," The Lancet, vol. 352, no. 9142, pp. 1725-1730, 1998.

[3] L. Zhang, B. Ramratnam, K. Tenner-Racz et al., "Quantifying residual HIV-1 replication in patients receiving combination antiretroviral therapy," The New England Journal of Medicine, vol. 340, no. 21, pp. 1605-1613, 1999.

[4] A. Carr, K. Samaras, A. Thorisdottir, G. R. Kaufmann, D. J. Chisholm, and D. A. Cooper, "Diagnosis, prediction, and natural course of HIV-1 protease-inhibitor-associated lipodystrophy, hyperlipidaemia, and diabetes mellitus: a cohort study," The Lancet, vol. 353, no. 9170, pp. 2093-2099, 1999.

[5] M. Harrington and C. C. J. Carpenter, "Hit HIV-1 hard, but only when necessary," The Lancet, vol. 355, no. 9221, pp. 2147-2152, 2000.

[6] V. A. Johnson, F. Brun-Vezinet, and B. Clotet, "Update of the drug resistance mutations in HIV-1: 2004, Top," HIV Med, vol. 12, pp. 119-124, 2004.

[7] F. Maggiolo, M. Airoldi, A. Callegaro et al., " $\mathrm{CD} 4$ cell-guided scheduled treatment interruptions in HIV-infected patients with sustained immunologic response to HAART," AIDS, vol. 23, no. 7, pp. 799-807, 2009.

[8] J. Lisziewicz, E. Rosenberg, J. Lieberman et al., "Control of HIV despite the discontinuation of antiretroviral therapy," The New England Journal of Medicine, vol. 340, no. 21, pp. 1683-1684, 1999.

[9] J. Anaworanich, R. Nuesch, M. L. Braz et al., "CD4 guided scheduled treatment interruption compared to continuous therapy: results of the staccato trial," Lancet, vol. 368, pp. 459465, 2006.

[10] M. L. G. Fernández, P. Rivas, M. Molina, R. Garcia, and M. De Górgolas, "Long-term follow-up of asymptomatic HIV-infected patients who discontinued antiretroviral therapy," Clinical Infectious Diseases, vol. 41, no. 3, pp. 390-394, 2005.

[11] F. Maggiolo, D. Ripamonti, G. Gregis, G. Quinzan, A. Callegaro, and F. Suter, "Effect of prolonged discontinuation of successful antiretroviral therapy on CD4 T cells: A controlled, prospective trial," AIDS, vol. 18, no. 3, pp. 439-446, 2004.

[12] B. Hirschel and T. Flanigan, "Is it smart to continue to study treatment interruptions?” AIDS, vol. 23, no. 7, pp. 757-759, 2009.

[13] L. Ruiz, R. Paredes, G. Gómez et al., "Antiretroviral therapy interruption guided by CD 4 cell counts and plasma HIV-1 RNA levels in chronically HIV-1-infected patients," AIDS, vol. 21, no. 2, pp. 169-178, 2007.

[14] M. M. Hadjiandreou, R. Conejeros, and D. I. Wilson, "Longterm HIV dynamics subject to continuous therapy and structured treatment interruptions," Chemical Engineering Science, vol. 64, no. 7, pp. 1600-1617, 2009.

[15] F. Lori, R. Maserati, A. Foli, E. Seminari, J. Timpone, and J. Lisziewicz, "Structured treatment interruptions to control HIV1 infection," The Lancet, vol. 355, no. 9200, pp. 287-288, 2000.

[16] C. Danel, R. Moh, A. Minga et al., "CD4-guided structured antiretroviral treatment interruption strategy in HIV-infected adults in west Africa (Trivacan ANRS 1269 trial): a randomised trial," The Lancet, vol. 367, no. 9527, pp. 1981-1989, 2006.

[17] DART trial team, "Fixed duration interruptions are inferior to continuous treatment in african adults starting therapy with CD4 cell counts $<200$ cells/ $\mu$ l," AIDS, vol. 22, pp. 237-247, 2008.

[18] W. M. EL-Sadr, J. D. Lundgren, J. D. Neaton et al., "CD4+ countguided interruption of antiretroviral treatment. the strategies for management of antiretro-viral therapy study group," The New England Journal of Medicine, vol. 355, pp. 2283-2296, 2006.

[19] G. A. Hardy, N. Imami, M. R. Nelson et al., "A phase I, randomized study of combined IL-2 and therapeutic immunisation with antiretroviral therapy," Journal of Immune Based Therapies and Vaccines, vol. 5, no. 1, p. 6, 2007.

[20] A. S. Perelson and P. W. Nelson, "Mathematical Analysis of HIV1 Dynamics in Vivo," SIAM Review, vol. 41, no. 1, pp. 3-44, 1999.

[21] M. A. Nowak and R. M. May, Virus Dynamics: Mathematics Principles of Immunology and Virology, Oxford University Press, New York, NY, USA, 2000.

[22] Y. Xiao, H. Miao, S. Tang, and H. Wu, "Modeling antiretroviral drug responses for HIV-1 infected patients using differential equation models," Advanced Drug Delivery Reviews, vol. 65, no. 7, pp. 940-953, 2013.

[23] S. M. Blower, H. B. Gershengorn, and R. M. Grant, "A tale of two futures: HIV and antiretroviral therapy in San Francisco," Science, vol. 287, pp. 650-654, 2007.

[24] L. Rong, Z. Feng, and A. S. Perelson, "Emergence of HIV1 drug resistance during antiretroviral treatment," Bulletin of Mathematical Biology, vol. 69, no. 6, pp. 2027-2060, 2007.

[25] S. Bonhoeffer, M. Rembiszewski, G. M. Ortiz, and D. F. Nixon, "Risks and benefits of structured antiretroviral drug therapy interruptions in HIV-1 infection," AIDS, vol. 15, pp. 2313-2322, 2000

[26] B. M. Adams, H. T. Banks, H. Kwon, and H. T. Tran, "Dynamic multidrug therapies for HIV: optimal and STI control approaches," Mathematical Biosciences and Engineering, vol. 1, no. 2, pp. 223-241, 2004.

[27] E. S. Rosenberg, M. Davidian, and H. T. Banks, "Using mathematical modeling and control to develop structured treatment interruption strategies for HIV infection," Drug and Alcohol Dependence, vol. 88, no. 2, pp. S41-S51, 2007.

[28] K. Y. Park, H. B. Chung, and C. Chung, "Analysis of treatment for HIV-infected patients considering CD4 T cell count in STI," in Proceedings of the 2006 SICE-ICASE International Joint Conference, pp. 5247-5252, Busan, South Korea, October 2006.

[29] S. Y. Tang, Y. N. Xiao, N. Wang et al., "Piecewise HIV virus dynamic model with CD4+ T cell count-guided therapy: I," Journal of Theoretical Biology, vol. 308, pp. 123-134, 2012.

[30] B. Tang, Y. N. Xiao, A. C. Robert et al., "Piecewise virus-immune dynamic model with HIV-1 RNA-guided therapy," Journal of Theoretical Biology, vol. 377, pp. 36-46, 2015.

[31] B. Tang, Y. Xiao, and J. Wu, "A piecewise model of virusimmune system with two thresholds," Mathematical Biosciences, vol. 278, pp. 63-76, 2016.

[32] B. Tang, Y. Xiao, S. Sivaloganathan, and J. Wu, "A piecewise model of virus-immune system with effector cell-guided therapy," Applied Mathematical Modelling: Simulation and Computation for Engineering and Environmental Systems, vol. 47, pp. 227-248, 2017.

[33] N. L. Komarova, E. Barnes, P. Klenerman, and D. Wodar, "Boosting immunity by antiviral drug therapy: A simple relationship among timing, efficacy, and success," Proceedings of the National Acadamy of Sciences, vol. 100, pp. 1855-1860, 2003. 
[34] H. Shu, L. Wang, and J. Watmough, "Sustained and transient oscillations and chaos induced by delayed antiviral immune response in an immunosuppressive infection model," Journal of Mathematical Biology, vol. 68, no. 1-2, pp. 477-503, 2014.

[35] A. Filippov, Differential Equations with Discontinuous RightHand Sides, Kluwer, Dordrecht, The Netherlands, 1988.

[36] Y. N. Xiao, T. T. Zhao, and S. Y. Tang, "Dynamics of an infectious diseases with media/psychology induced non-smooth incidence," Mathematical Biosciences and Engineering, vol. 10, no. 2, pp. 445-461, 2013.

[37] A. Wang and Y. Xiao, "A Filippov system describing media effects on the spread of infectious diseases," Nonlinear Analysis: Hybrid Systems, vol. 11, no. 1, pp. 84-97, 2014.

[38] B. Tang, Y. Xiao, S. Tang, and R. Cheke, "A feedback control model of comprehensive therapy for treating immunogenic tumours," International Journal of Bifurcation and Chaos, vol. 26, no. 3, 2016.

[39] V. L. Utikin, J. Guldner, and J. X. Shi, Sliding mode control in Electro-Mechanical system, Taylor \& Fraacis Group, 2nd edition, 2009. 


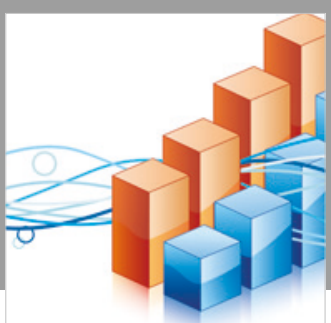

Advances in

Operations Research

\section{-n-m}
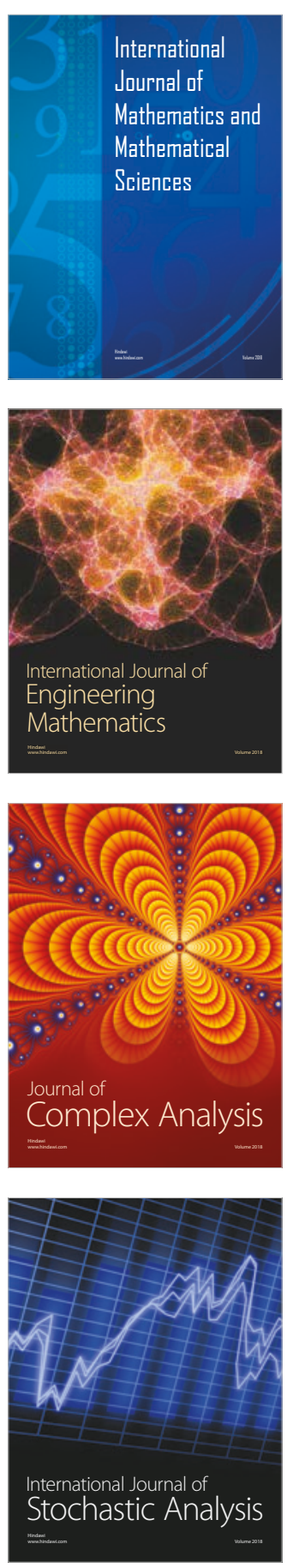
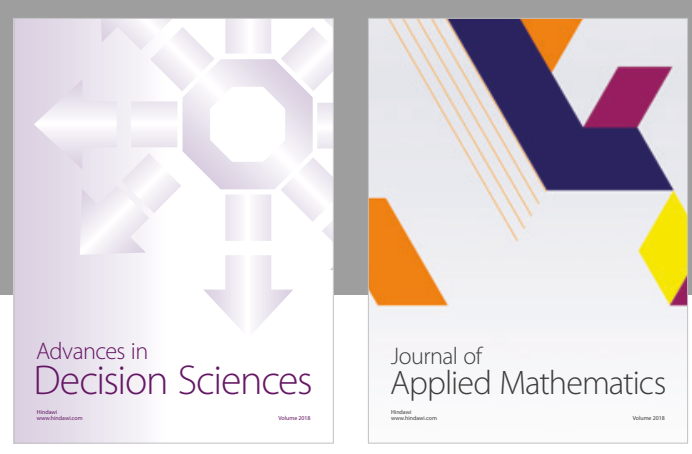

Journal of

Applied Mathematics
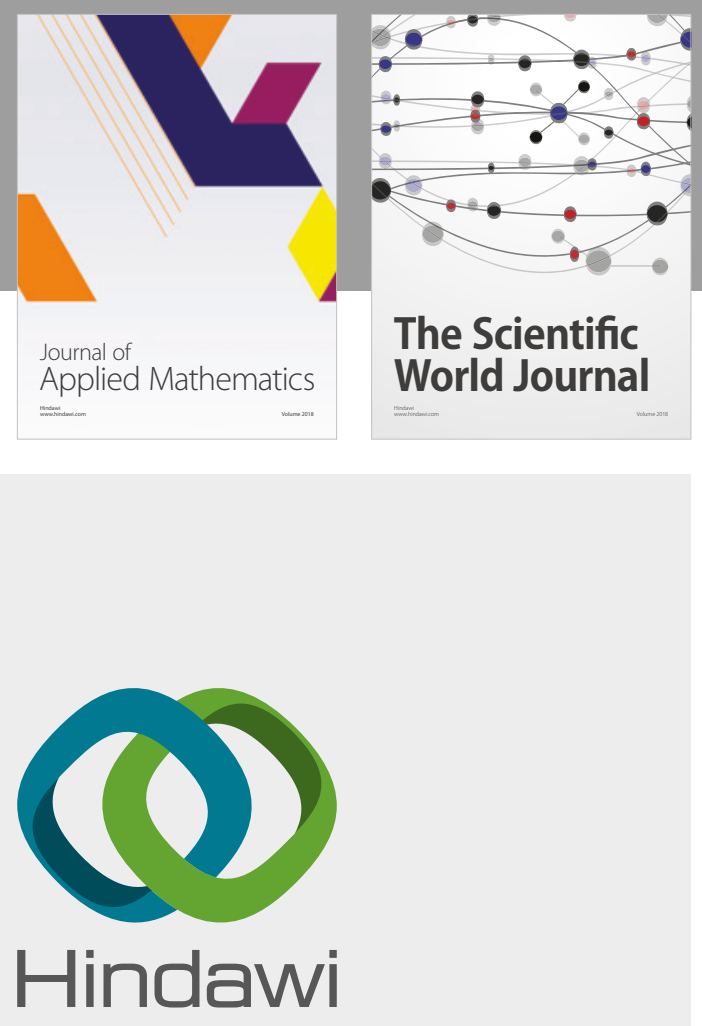

Submit your manuscripts at

www.hindawi.com

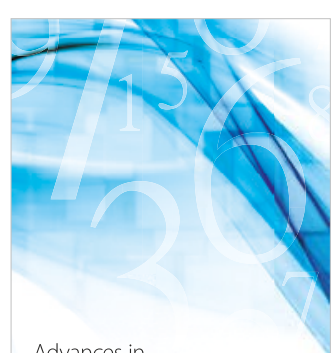

Advances in
Numerical Analysis
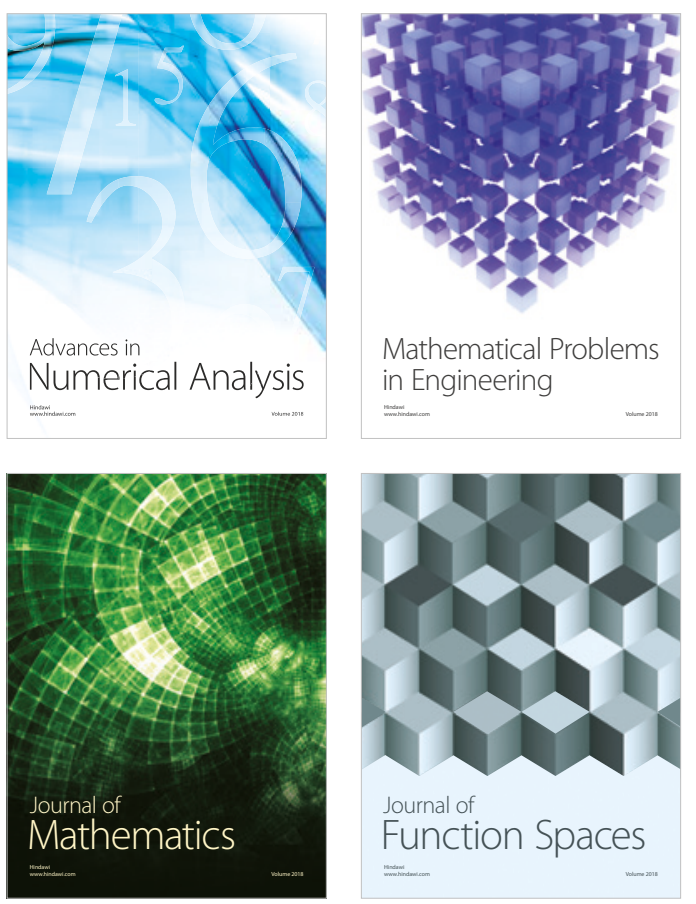

Mathematical Problems in Engineering

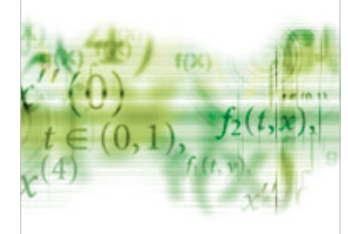

International Journal of

Differential Equations

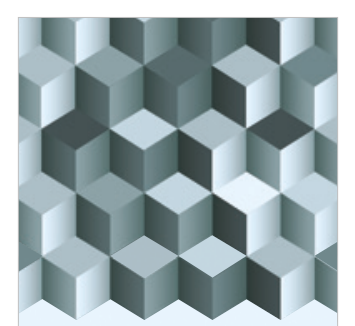

Journal of

Function Spaces

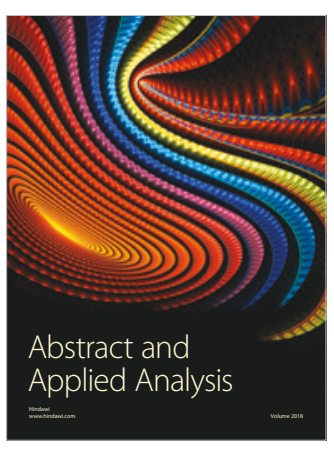

The Scientific

World Journal

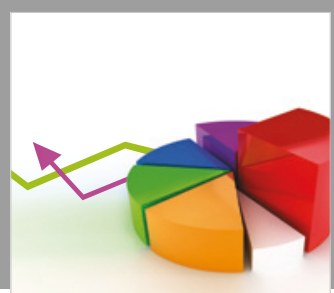

Journal of

Probability and Statistics
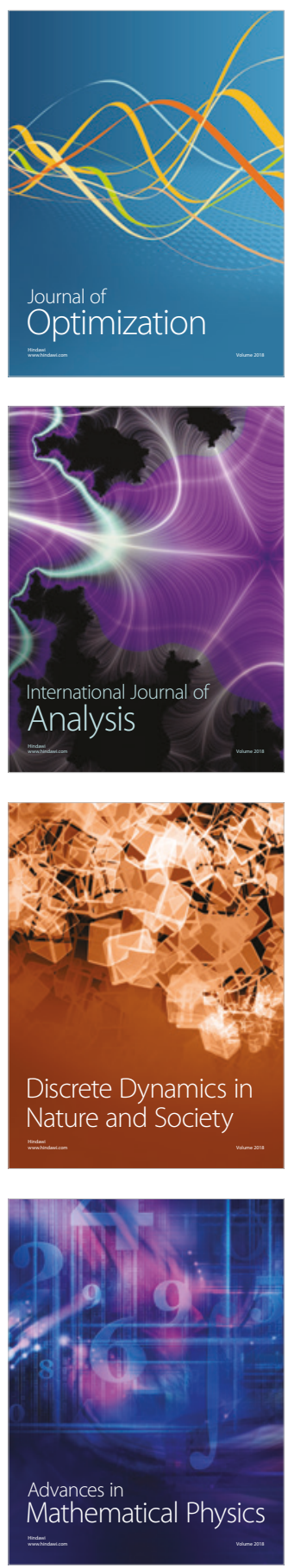